\title{
Orthopyroxenite hosted chromitite veins anomalously enriched in platinum-group minerals from the Havana-Matanzas Ophiolite, Cuba
}

\author{
Vetas de cromitita en ortopiroxenita anómalamente enriquecidas en minerales del grupo del platino \\ de la ofiolita Habana-Matanzas, Cuba
}

Júlia Farré-de-Pablo ${ }^{1, *}$, Núria Pujol-Solà ${ }^{1}$, Harlison Torres-Herrera ${ }^{2}$, Thomas Aiglsperger ${ }^{3}$, José María González-Jiménez $^{4}$, Angélica Isabel Llanes-Castro ${ }^{5}$, Antonio Garcia-Casco ${ }^{4,6}$, Joaquín A. Proenza ${ }^{1}$

\begin{abstract}
${ }^{1}$ Departament de Mineralogia, Petrologia i Geologia Aplicada. Facultat de Ciències de la Terra, Universitat de Barcelona. G/ Martí i Franquès, s/n, 08028, Barcelona, Spain.

2 Fundación Universitaria del Area Andina - Sede Valledupar. Transv 22 Bis 4-105, Valledupar, Colombia.
\end{abstract}

${ }^{3}$ Department of Civil Engineering and Natural Resources, Luleå University of Technology. SE 97187, Luleå, Sweden.

${ }^{4}$ Departamento de Mineralogía y Petrología, Facultad de Ciencias, Universidad de Granada. Avda. Fuentenueva, s/n, 18002, Granada, Spain.

${ }^{5}$ Departamento de Petrología y Mineralogía, Instituto de Geología y Paleontología. Vía Blanca 1002, San Miguel del Padrón, Cuba.

${ }^{6}$ Instituto Andaluz de Ciencias de la Tierra (CSIC-UGR). Avda. de las Palmeras 4, E-18100 Armilla, Granada, Spain.

* Corresponding author: (J. Farré de Pablo) jfarredepablo@gmail.com

\begin{abstract}
How to cite this article:
Farré-de-Pablo, J., Pujol-Solà, N., TorresHerrera, H., Aiglsperger, T., GonzálezJiménez, J. M., Llanes-Castro, A. I., Garcia-Casco, A., Proenza, J. A., 2020, Orthopyroxenite hosted chromitite veins anomalously enriched in platinum-group minerals from the Havana-Matanza Ophiolite, Cuba: Boletín de la Sociedad Geológica Mexicana, 72 (3), Al10620. http://dx.doi. org/10.18268/BSGM2020v72n3a110620
\end{abstract}

Manuscript received: May 11, 2020

Corrected manuscript received: June 5,2020

Manuscript accepted: June 10,2020

Peer Reviewing under the responsibility of Universidad Nacional Autónoma de México.

\begin{abstract}
The Havana-Matanzas Ophiolite contains one of the few examples of ophiolitic platinum group minerals (PGM)-rich chromitites associated with orthopyroxenites in the mantle section of ophiolitic complexes. The chromitites occur as veins hosted by orthopyroxenite bands within mantle peridotites. The peridotites are mostly harzburgites and their accessory chromite shows high- $\mathrm{Al}$ compositions $(\mathrm{Cr} \#[\mathrm{Cr} /(\mathrm{Cr}+\mathrm{Al})$, atomic ratio $=0.39-0.50$ ), which are typical of spinels in abyssal peridotites. Conversely, chromite from the chromitite veins and their host orthopyroxenite are high- $\mathrm{Cr}(\mathrm{Cr} \#$ $=0.72-0.73$ and $0.62-0.69$, respectively), with lower $\mathrm{Mg} \#\left[\mathrm{Mg} /\left(\mathrm{Mg}+\mathrm{Fe}^{2+}\right)\right.$, atomic ratio]. This suggests that both the chromitite and the orthopyroxenite formed from melts with boninitic affinity. The abundant PGM inclusions found in the chromitites are mainly Os-rich laurite grains, which is also characteristic of chromitites formed from magmas with boninitic affinity. Therefore, we propose that the chromitite veins and the orthopyroxenite bands probably formed contemporaneously in the fore-arc setting of an intra-oceanic arc during subduction. The chromitite-orthopyroxenite pair of the Havana-Matanzas Ophiolite could form after the reaction of a Si-rich melt with boninitic affinity and mantle harzburgite, with the orthopyroxenite bands preserving fingerprints of the infiltration of boninitic-affinity melts within the mantle. The small volume of forming chromitite could maximize the efficiency for the mechanical collection of the PGM forming in the parental melt of these rocks, resulting in the anomalous enrichment of primary PGM in the chromitites.
\end{abstract}

Keywords: chromitite, Cuba, ophiolite, orthopyroxenite, PGM.

\section{RESUMEN}

La ofiolita de Habana-Matanzas contiene uno de los pocos ejemplos de cromititas ricas en minerales del grupo del platino (MGP) asociadas a ortopiroxenitas de la sección mantélica de complejos ofiolíticos. Las cromititas ocurren como venas encajadas en bandas de ortopiroxenita dentro de la peridotita mantélica. Las peridotitas son mayoritariamente harzburgitas con cromita accesoria rica en $\mathrm{Al}$ (\#Cr $[\mathrm{Cr} /(\mathrm{Cr}+\mathrm{Al})$, cociente atómico $]=0.39-0.50)$, lo cual es típico de espinelas en peridotitas abisales. Por otro lado, la cromita perteneciente a las venas de cromitita y a la ortopiroxenita encajante es rica en $\mathrm{Cr}(\# \mathrm{Cr}=0.72-0.73$ y $0.62-0.69$, respectivamente) y con bajo \#Mg $\left[\mathrm{Mg} /\left(\mathrm{Mg}+\mathrm{Fe}^{2+}\right)\right.$, cociente atómico]. Esto sugiere que tanto las venas de cromitita como la ortopiroxenita se formaron a partir de fundidos de afinidad boninítica. Las abundantes inclusiones de MGP encontradas en las cromititas son principalmente granos de laurita ricos en $\mathrm{Os}$, lo cual también es propio de cromititas formadas a partir de magmas con afinidad boninítica. Por lo tanto, proponemos que las venas de cromitita y las bandas de ortopiroxenitas se formaron contemporáneamente en un contexto de ante-arco en un arco intra-oceánico durante el proceso de subducción. El conjunto cromitita-ortopiroxenita de la ofiolita de HabanaMatanzas se formó por la reacción de fundidos ricos en Si con afinidad boninitica y la harzburgita mantélica. Las bandas de ortopiroxenitas sería la huella química que habrían dejado estos fundidos boniníticos al infiltrarse por el manto. El volumen reducido de las cromititas que se formaron maximizó la eficiencia del proceso de recolección mecánica de los MGP que se formaban en el fundido parental, dando lugar al enriquecimiento de MGP primarios como inclusiones en las cromititas.

Palabras clave: cromititas, Cuba, MGP, ofiolita, ortopiroxenita. 


\section{Introduction}

Chromitite bodies from the mantle section of ophiolites are frequently enveloped by dunites hosted in mantle harzburgites (e.g., Leblanc and Nicolas, 1992; González-Jiménez et al., 2014, and references therein). The formation of these mantle chromitites is commonly associated with basaltic melts reaching the mantle-crust transition zone of the oceanic lithosphere through percolation processes and their consequent melt-rock reaction in the upper mantle (e.g., Arai and Miura, 2016, and references therein). Typically, high-Al chromitites $(\mathrm{Cr} \#[\mathrm{Cr} /(\mathrm{Cr}+\mathrm{Al})$, atomic ratio $]<0.60)$ are located near the Moho Transition Zone, while high-Cr ( $\mathrm{Cr} \#>0.60)$ occur deeper in the upper mantle levels from the ophiolitic sequence (e.g., Proenza et al., 1999). High-Al mantle chromitites are usually enriched in $\mathrm{TiO}_{2}(\sim 0.5$ wt. $\%)$ and depleted in platinum group elements (PGE: Os, $\mathrm{Ru}, \mathrm{Ir}, \mathrm{Pt}, \mathrm{Pd}, \mathrm{Rh}$ ), whereas high-Cr chromitites are depleted in $\mathrm{TiO}_{2}(<0.25$ wt. \%) and enriched in PGE (e.g., Gervilla et al., 2005; González-Jiménez, 2011a). The mineralogical expression of PGE enrichment is the presence of abundant micro inclusions of platinum group minerals (PGM) in chromite, especially members of the solid solution series laurite $\left(\mathrm{RuS}_{2}\right)$ - erlichmanite $\left(\mathrm{OsS}_{2}\right)$, Os-Ir alloys and Ir-sulfarsenides (e.g., Zhou et al., 1996; Ahmed and Arai, 2002).

Even if chromitites are globally associated with dunite bodies hosted in harzburgite, chromitites are locally associated with orthopyroxenites, such as in the Bay of Islands ophiolites in Newfoundland (Canada) (Bédard and Hebert, 1998), in the northern part of the Oman ophiolite (Ahmed and Arai, 2002), and the Ouen Island in New Caledonia (González-Jiménez et al., 2011b). In the Bay of Islands ophiolites, high-Cr chromitite replaces orthopyroxenite, interlayered with peridotite (mostly dunite), along its rim. According to Bédard and Hebert (1998), the composition of chromite from their studied chromitites depends more on the nature of the minerals being assimilated than on the composition of the magma. In Oman, high-Cr chromitite pods with unusually high IPGE (Os, Ru, Ir) contents are described in the deep mantle section related to orthopyroxenite bands within harzburgite (Ahmed and Arai, 2002). Their origin is linked to trapped refractory mafic melts derived from high-degree partial melting of peridotite in a suprasubduction zone (SSZ) environment (Ahmed and Arai, 2002). In New Caledonia, the chromitites from Ouen Island formed from small-volume hydrous silicate melts and are high-Al and very PGE-rich. They are associated to orthopyroxenite pockets within wehrlitic units (González-Jiménez et al., 2011b). Another good example of the chromitite-orthopyroxenite association is from the subcontinental mostly lherzolitic massif of Ronda (Spain), where high-Cr and high-Al chromitites are spatially associated with clinopyroxenites and orthopyroxenites (Gervilla et al., 2019). Here, chromitite occurs as entwined and contorted veins replacing pyroxenites with a clear correlation between the composition of chromite grains and the type of pyroxene relicts found in the them, orthopyroxene in high-Cr versus clinopyroxene in high-Al chromitite, which would reflect the $\mathrm{Cr}_{2} \mathrm{O}_{3} / \mathrm{Al}_{2} \mathrm{O}_{3}$ ratio of the reacting mafic rock (González-Jiménez et al., 2013).

In this work, we study an unprecedented case of chromitite veins hosted in orthopyroxenite bands in the mantle tectonites from the Havana-Matanzas Ophiolite, in Cuba. These chromitites are characterized by being extremely rich in platinum-group mineral (PGM) inclusions. We provide detailed petrographic and geochemical description of the host peridotites, orthopyroxenites, and chromitite veins and their PGM inclusions in order to shed some light on the genesis of the chromitites and the possible genetic relations with their orthopyroxenite host.

\section{Geological setting}

The northern margin of the island of Cuba contains the largest and more abundant ophiolite bodies of the Caribbean region, extending ca. $1000 \mathrm{~km}$ from SE to NW (Figure 1A; Iturralde-Vinent, 1996, 1998; Garcia-Casco et al., 2006; Lewis et al., 2006; Iturralde-Vinent et al., 2016). These ophiolites are 
part of the Cuban orogenic belt, and represent slices of oceanic lithosphere ( 136 to $70 \mathrm{Ma}$; RojasAgramonte et al., 2010) obducted onto the North American continental paleo-margin in Late Cretaceous to mid Eocene times during the collision of the Caribbean volcanic arc with the Jurassic-Cretaceous passive margins of the continental Maya block and the Bahamas platform (Iturralde-Vinent, 1996, 1998; Garcia-Casco et al., 2008; Iturralde-Vinent et al., 2008, 2016). The ophiolite assemblages are highly tectonically dismembered, but sections of large volumes of mantle tectonites and a Moho Transition Zone (dunite, wehrlite, plagioclase- and clinopyroxene-bearing peridotites, sills and dikes of gabbro) associated with a crustal sequence (layered gabbros, mafic volcanic rocks and sediments) can be recognized. Field relations, petrography, mineral chemistry, and whole rock compositions indicate a predominance of oceanic lithosphere formed above subducting plates (i.e., SSZ-type ophiolites) (e.g., Proenza et al., 1999; Iturralde-Vinent et al., 2016). These SSZ ophiolites from the basement of the Pacific (Farallon)-derived Caribbean plate are intimately related to the origin and evolution of the Caribbean volcanic arc ( 135-70 Ma).

The Havana-Matanzas Ophiolite is located at the west-central part of the island (Figure 1A). It consists of a complete but tectonically dismembered ophiolitic sequence with a predominance of upper mantle rocks over crustal rocks (Llanes-Castro et al., 2015, and references therein). As part of a tectonic mélange, the Havana-Matanzas Ophiolite are tectonically imbricated with Mesozoic sedimentary rocks of the southern paleo-margin of North America, Cretaceous intra-oceanic volcanic arc units, and Late Cretaceous to Eocene synorogenic sedimentary sequences (Figure 1B; Llanes-Castro et al., 2015). The tectonic blocks/ slices of ophiolitic rocks crop out as bodies that extend up to $15-20 \mathrm{~km}$ long and 2-3 km wide, although the sequence may reach $4 \mathrm{~km}$ in thickness (Iturralde-Vinent, 1996). The reconstructed Havana-Matanzas Ophiolite sequence consists of a lower unit of upper mantle peridotites, the Moho Transition Zone, and a crustal section composed of massive (isotropic) gabbros, dolerites dykes, pillow lavas with island-arc tholeiite (IAT) and boninitc affinities, and volcano-sedimentary rocks on top (Fonseca et al., 1985; Kerr et al., 1999; Llanes-Castro et al., 2015). The mantle peridotites are plagioclase lherzolites and highly serpentinized harzburgites with porphyroclastic fabric (Fig. 1C), which contain ubiquitous orthopyroxenite veins and minor sub-concordant dunite layers (Lewis et al., 2006; Llanes-Castro et al., 2015).

The Havana-Matanzas Ophiolite contains a total of 10 chromitite occurrences (Llanes-Castro et al., 2015, and references therein). These chromitites occur as small bodies (around $0.5 \mathrm{~m}$ wide and $2 \mathrm{~m}$ long) with tabular to lenticular shapes. They are spatially related to mantle tectonites from the Moho Transition Zone and are enclosed in deformed serpentinized dunites within highly serpentinized harzburgite (Llanes-Castro et al., 2015). The majority of these chromitites are concordant with the foliation of their host peridotites. They display predominantly massive texture, but also nodular to disseminated textures at the margin of some chromitite bodies (Llanes-Castro et al., 2015). Chemically, they are classified as both high-Al $(\mathrm{Cr} \#=0.44-0.46)$ and high-Cr $(\mathrm{Cr} \#=0.65-0.74)$ chromitites (Llanes-Castro et al., 2015). According to these authors, the composition of the parental magmas of the high-Al chromitites had a back-arc basin basalt (BABB) affinity, and the composition of the high-Cr parental magmas had IAT affinity. This is also in accordance with the occurrence of platinum group mineral (PGM) inclusions, mainly laurite, in high-Cr chromitites, which indicates crystallization at high temperatures $\left(\sim 1300^{\circ} \mathrm{C}\right)$ typical of boninitic magmas.

Chromitites in Havana-Matanzas Ophiolite also occur as small veins within orthopyroxenite bands (Figure 1D to 1E). The orthopyroxenite bands are up to $1.5 \mathrm{~m}$ in thickness and are systematically emplaced in highly serpentinized harzburgites. The chromitite veins hosted by the pyroxenites are centimetric in thickness with a lateral extension of several decimeters. They display massive fabric (> $80 \mathrm{vol} . \%$ chromite) which tends to be disseminated (20-80 vol. \% chromite) at the contact with the pyroxenite (Figure 1E). 

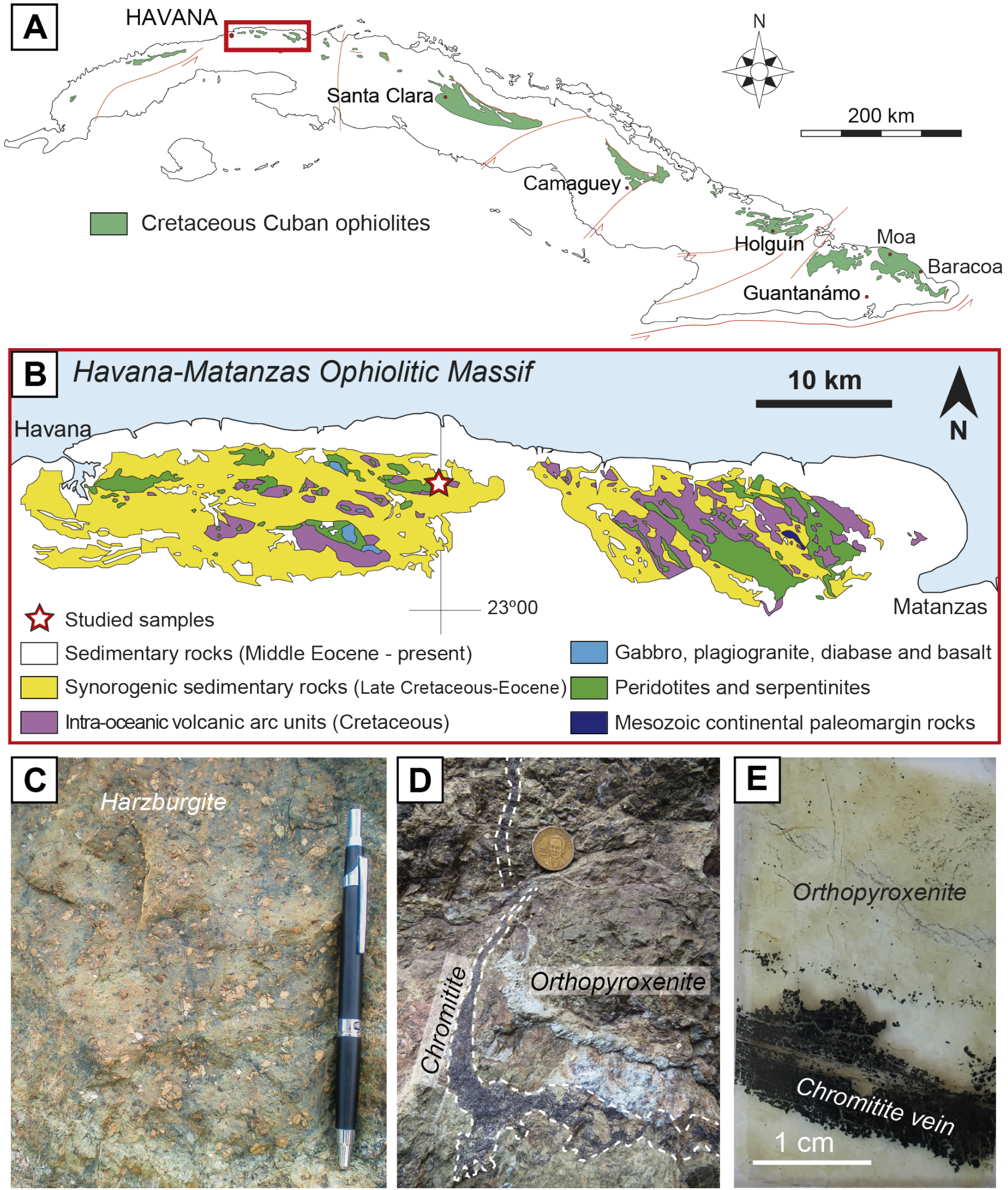

Figure 1 A: Geological sketch map showing location of the Cuban ophiolitic belt. The red rectangle marks the position of the HavanaMatanzas Ophiolitic Massif (modified after Iturralde-Vinent et al., 2016). B: Geological map of the Havana-Matanzas Ophiolitic Massif with the location of the studied samples (modified after Llanes-Castro et al., 2019). C: field photograph of harzburgite with porphyroclastic texture. D: field photograph of a chromitite vein and the host orthopyroxenite. E: thin section of a chromitite vein and the host orthopyroxenite. 


\section{Samples and analytical methods}

Samples of the chromitite veins and their host orthopyroxenite and peridotites were collected from the area indicated in Figure 1B, in the Havana-Matanzas Ophiolitic Massif. We prepared thin and thick polished sections and studied them under optical microscope and scanning electron microscope (SEM), using a Quanta 200 FEI XTE 325/D8395 equipped with an INCA Energy 250 EDS at the Centres Cientifics $i$ Tecnologics de la Universitat de Barcelona (CCiT-UB). Representative mineral composition of olivine, orthopyroxene, chromite, and platinum group minerals (PGM) were obtained using a JEOL JXA-8230 electron microprobe equipped with five WDS spectrometers and one EDS spectrometer at the CCiT-UB. The analytical conditions were $20 \mathrm{kV}$ accelerating voltage, $15 \mathrm{nA}$ beam current with spot diameter 1-5 $\mu \mathrm{m}$, and $20 \mathrm{~s}$ count time.

The calibration standards used for silicates and oxides were: hematite (Fe-LIF-Ka), rutile (Ti-PET-Ka), periclase (Mg-TAP-Ka), rhodonite (Mn-LIF-Ka), $\mathrm{Al}_{2} \mathrm{O}_{3}$ (Al-TAP-Ka), $\mathrm{Cr}_{2} \mathrm{O}_{3}$ (Cr-PET-Ka), diopside (Si-TAP-Ka), wollastonite (Ca-PET-Ka), albite (Na-TAP-Ka), and orthoclase (K-PET-La). The analytical conditions for the PGM were $15 \mathrm{kV}$ accelerating voltage, 10-20 nA beam current, $2 \mu \mathrm{m}$ spot diameter and $20 \mathrm{~s}$ count time. The calibration standards used were: native metals (Os-LIF-La, Ir-LIFL-La, Ru-PETJ-La, Rh-PETJ-La, Pt-LIFL-La, Pd-PETJ-L $\beta$, Co-LIFH-Ka， Cr-LIFH-Ka， Ni-LIFH-Ka), $\mathrm{FeS}_{2}$ (Fe-LIFH-Ka, S-PETJ-Ka), chalcopyrite (Cu-LIFL-Ka), and GaAs (As-TAPH- L $\beta$ ).

A representative chromitite vein sample $(\sim 300$ g) was processed in order to obtain heavy mineral concentrates. The sample was crushed, grinded and sieved into different fractions $(106-75 \mu \mathrm{m}$, 75-53 $\mu \mathrm{m}, 53-30 \mu \mathrm{m}$, and $<30 \mu \mathrm{m}$ ) that were processed at the Hydroseparation Laboratory of the University of Barcelona (www.hslab-barcelona. com). The resulting concentrates were mounted into thick sections which were polished and studied by means of field emission scanning electron microscopy (FE-SEM) using a JEOL JSM-7100 at the CGiT-UB.

\section{Petrography}

\subsection{HARZBURGITE}

Harzburgites hosting pyroxenite bands with chromitite veins consist of partially serpentinized olivine (65-75 vol.\%), orthopyroxene (25-35 vol.\%), clinopyroxene ( $<2$ vol. $\%$ ) and accessory chromian spinel $(<1$ vol. $\%)$. Their texture is characterized by plastic deformation of orthopyroxene porphyroclasts, typical of mantle tectonites deformed at high temperature. Olivine grains $(<2 \mathrm{~mm}$ in size) occur as partially serpentinized neoblasts that constitute the matrix of the rock (Figure 2A and 2B). Orthopyroxene porphyroclasts are up to $5 \mathrm{~mm}$ in size with rounded shapes (Figure 2A and $2 \mathrm{~B}$ ). They are locally replaced by olivine in embayments along their boundaries (Figure 2B). Clinopyroxene grains are scarce and generally altered to amphibole. The accessory chromian spinel grains (referred hereafter as chromite, for convenience) are subhedral to anhedral with size ranging between $10 \mu \mathrm{m}$ and $1 \mathrm{~mm}$. Locally, they are partially or completely altered to ferrian chromite and magnetite plus chlorite. Mesh and bastite textures were produced during pseudomorphization of previous minerals by serpentine group minerals, mainly lizardite (Figure 2A).

\subsection{ORTHOPYROXENITE BANDS}

Orthopyroxenite bands within harzburgite mainly consist of orthopyroxene (80-90 vol. \%) and olivine (up to $18 \mathrm{vol} . \%)$, with minor clinopyroxene $(<3$ vol. \%) and accessory chromite ( $<2$ vol. $\%)$. Generally, they display equigranular texture with orthopyroxene grains up to $5 \mathrm{~mm}$ in size and olivine and clinopyroxene grains around $0.5 \mathrm{~mm}$ in size (Figure $2 \mathrm{C}$ to $2 \mathrm{D})$. Accessory chromite grains are euhedral and less than $0.2 \mathrm{~mm}$ in size. They are hosted both in orthopyroxene and olivine grains (Figure 2D). 

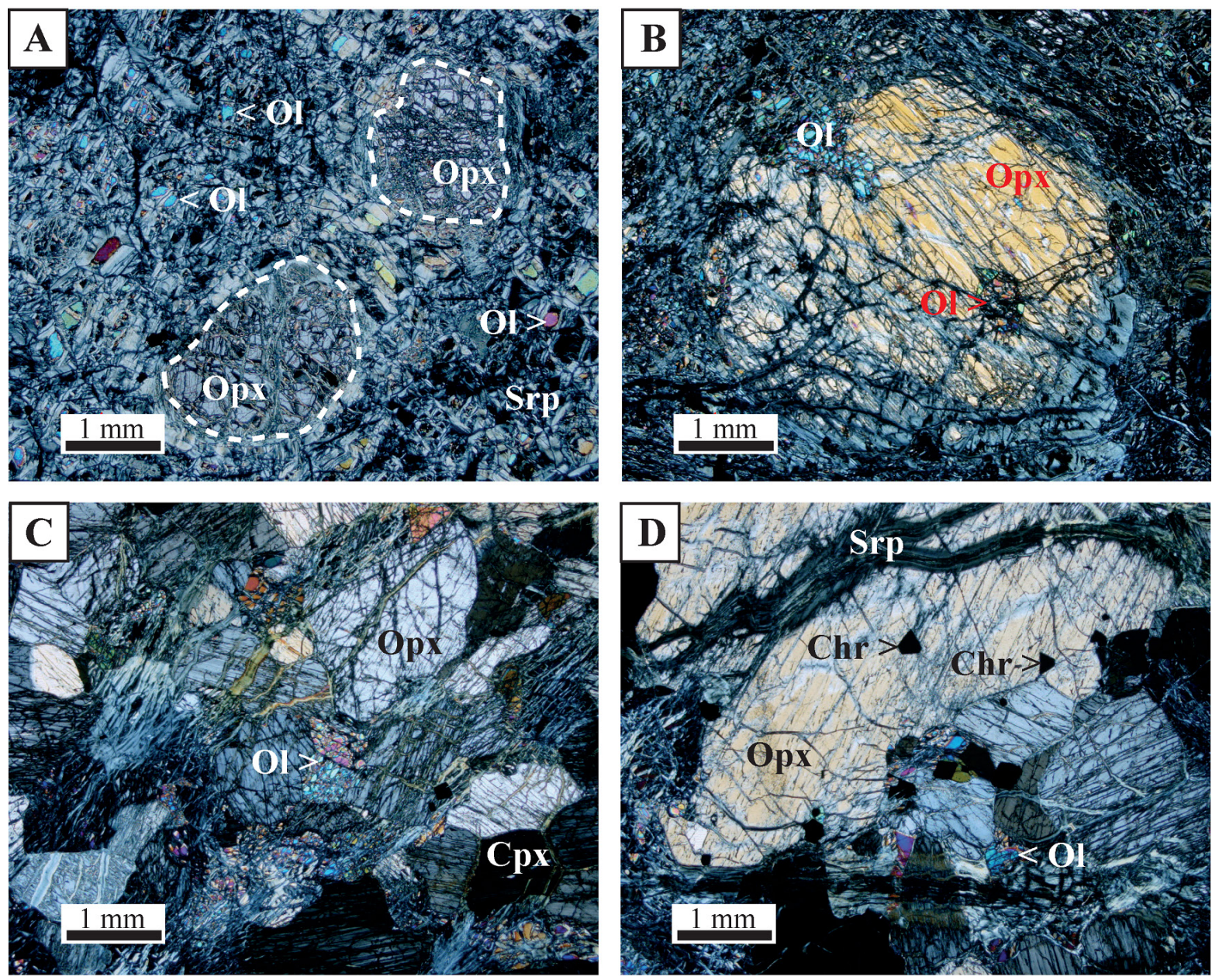

Figure 2 Optical microscope images of harzburgite (A, B) and orthopyroxenite (C, D). A: orthopyroxene porphyroclasts in a serpentinized matrix of olivine from a harzburgite sample. B: orthopyroxene porphyroclast with embayments filled by olivine surrounded by a serpentinized matrix of olivine neoblasts in harzburgite. C: orthopyroxene, olivine and clinopyroxene grains showing granoblastic texture in orthopyroxenite. D: accessory chromite associated with both orthopyroxene and olivine in orthopyroxenite. Opx = orthopyroxene; OI = olivine; Srp = serpentine; $\mathrm{Cpx}=$ clinopyroxene; $\mathrm{Chr}=$ chromite.

\subsection{GHROMITITE VEINS}

The chromitite veins in the orthopyroxenite bands show massive textures (> 80 vol. \% chromite) with euhedral to subhedral chromite grains which are locally intensely fractured (Figure $3 \mathrm{~A}$ and $3 \mathrm{~B}$ ). Serpentine fills the fractures and the interstitial spaces between chromite grains. The chromite grains are homogeneous, with no alteration rims, and their sizes vary from larger grains $(<0.5 \mathrm{~mm})$ in the middle of the veins, to smaller grains (down to $50 \mu \mathrm{m}$ ) towards the contact with the orthopyroxenite. Chromite grains locally show embayment at the contact with the host orthopyroxenite (Figure 3C), and may host silicate inclusions and PGM. The silicate inclusions are around $10 \mu \mathrm{m}$ in size with euhedral to round shapes, and they are often aligned along trails crossing the chromite grains (Figures 3D to 3F). These inclusions consist of phlogopite, olivine and orthopyroxene that, in most cases, have been altered to serpentine and bastite, respectively. PGM inclusions are very abundant in the chromitite veins, which is one of their most remarkable characteristics.

\subsection{PGM IN THE GHROMITITE VEINS}

Platinum group minerals (PGM) were identified both in situ in unaltered chromite grains from the chromitite veins (3 PGM grains; Figure 4A and $4 \mathrm{~B})$ and in the heavy mineral concentrates from the processed chromitite vein samples (17 PGM 

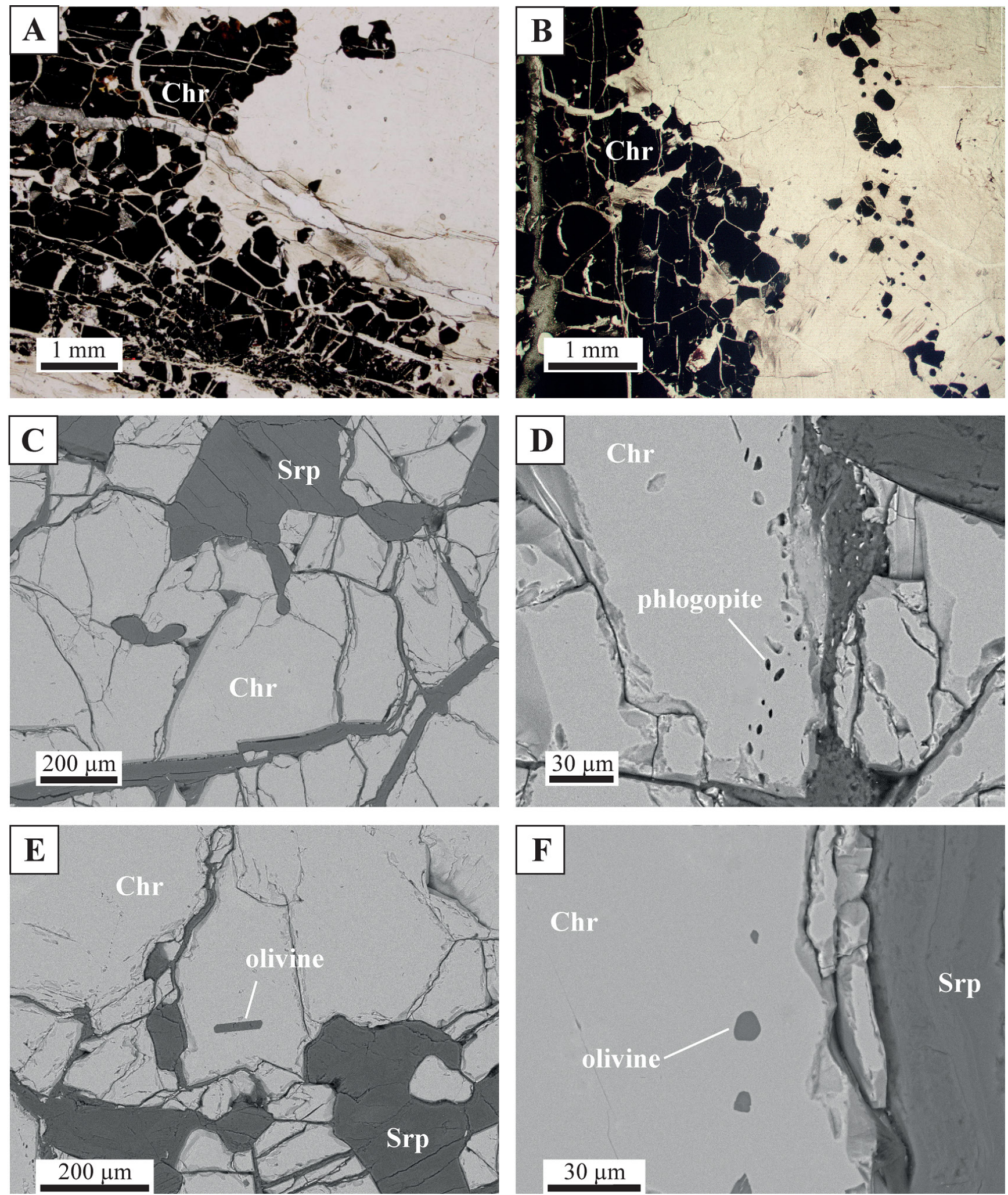

Figure 3 Optical microscope (A, B) and BSE (C-F) images of the chromitite veins. A and B: contact of the chromitite vein with the host orthopyroxenite. C: chromite grains at the contact with the host rock. D: trail of subhedral inclusions (olivine, clinopyroxene and serpentine) across chromite grains. E: single elongated olivine inclusion within chromite. F: trail of rounded inclusions (olivine and serpentine). $\mathrm{Chr}=$ chromite; Srp = serpentine. 

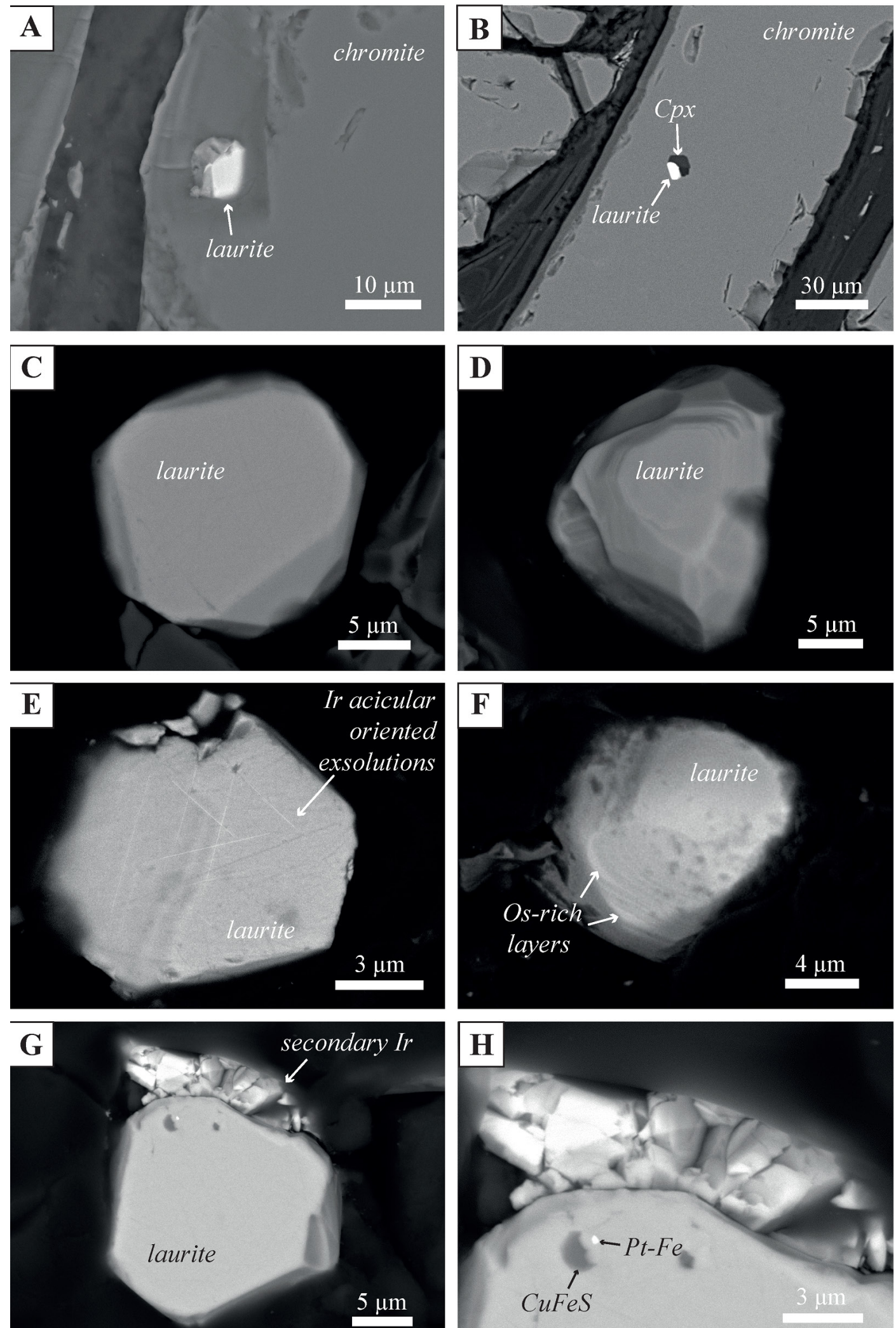

Figure 4 BSE images of PGM found in the chromitite veins from Havana-Matanzas Ophiolite. A: in situ grain of laurite within a chromite grain. B: in situ laurite grain together with clinopyroxene (Cpx). C: homogeneous euhedral Os-rich laurite. D: homogeneous subhedral Os-rich laurite with crystal growth bands. E: euhedral laurite grain with acicular oriented exsolutions of Ir along crystallographic planes. F: laurite crystal with oscillatory zoning. G: euhedral laurite with nanometric inclusions and Ir on the surface. H: detail of the nanometric inclusions of the laurite crystal shown in G. 

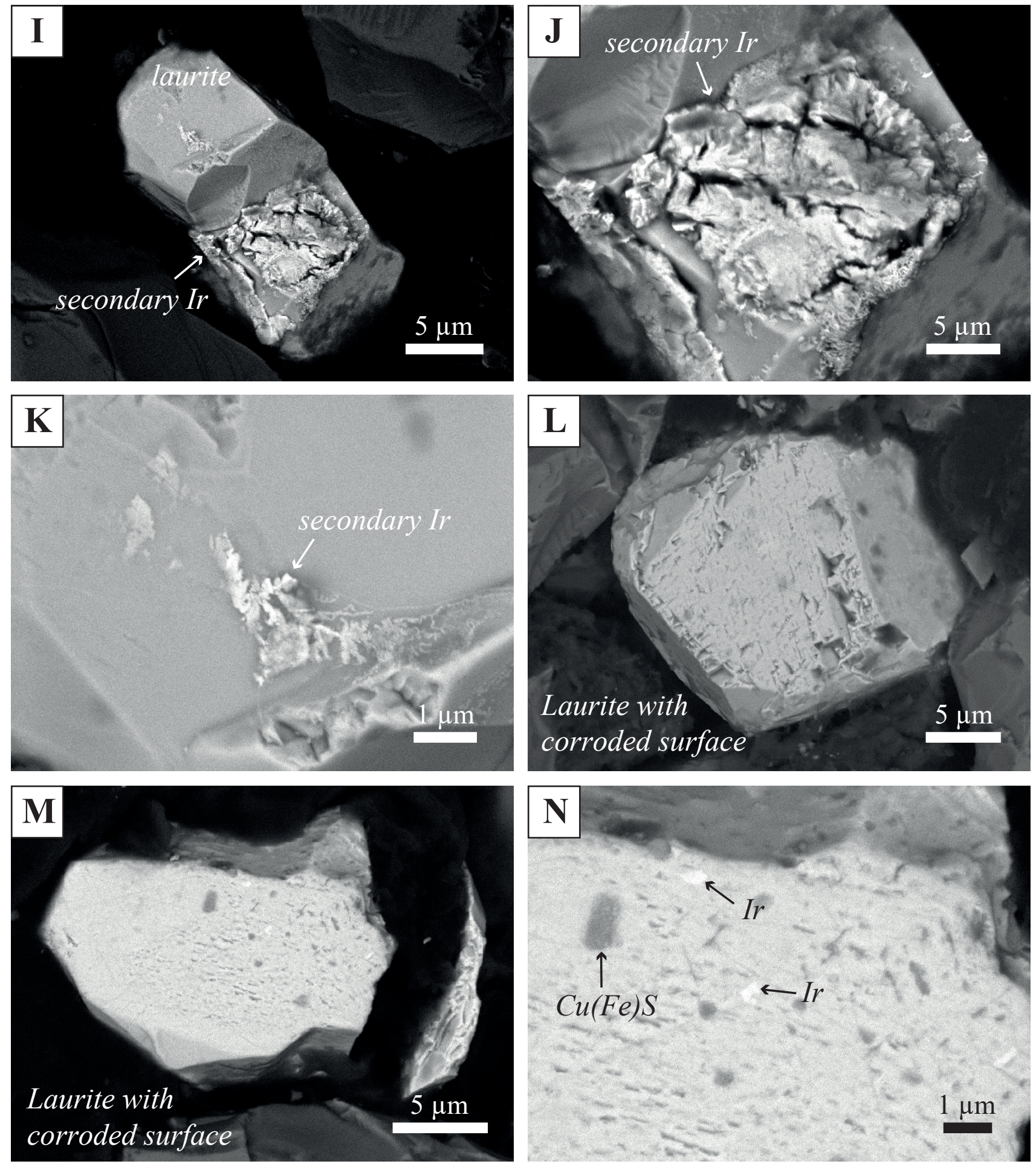

Figure 4 (Continuation) BSE images of PGM found in the chromitite veins from Havana-Matanzas Ophiolite. I: subhedral Os-rich laurite with overgrowth of Ir on the surface. J: detail of I showing fibrous texture of Ir. K: detail of I showing aggregate almost dendritic texture of Ir. L: euhedral laurite with corroded surface. M: subhedral laurite with corroded surface and nanoinclusions. N: detail of the nanometric inclusions of laurite shown in $\mathrm{M}$. 
Table 1. Representative electron microprobe analyses of olivine from harzburgites $(\mathrm{Hz})$ and orthopyroxenites (Opyr) from the HavanaMatanzas Ophiolite. $\mathrm{Fo}=\mathrm{Mg} /\left(\mathrm{Mg}+\mathrm{Fe}^{2+}\right)$ x 100.

\begin{tabular}{|c|c|c|c|c|c|c|c|c|c|c|}
\hline Rock type & $\mathrm{Hz}$ & $\mathrm{Hz}$ & $\mathrm{Hz}$ & $\mathrm{Hz}$ & $\mathrm{Hz}$ & Opyr & Opyr & Opyr & Opyr & Opyr \\
\hline $\mathrm{SiO}_{2}$ (wt.\%) & 40.61 & 40.78 & 40.99 & 40.70 & 41.21 & 40.56 & 40.65 & 40.39 & 40.52 & 40.26 \\
\hline $\mathrm{TiO}_{2}$ & $b d l$ & $b d l$ & $b d l$ & $b d l$ & $b d l$ & $b d l$ & $b d l$ & $b d l$ & $b d l$ & 0.01 \\
\hline $\mathrm{Al}_{2} \mathrm{O}_{3}$ & $b d l$ & $b d l$ & $b d l$ & 0.01 & 0.02 & 0.01 & $b d l$ & $b d l$ & 0.02 & 0.01 \\
\hline $\mathrm{V}_{2} \mathrm{O}_{3}$ & 0.01 & 0.01 & $b d l$ & $b d l$ & $b d l$ & 0.01 & 0.01 & 0.02 & $b d l$ & 0.02 \\
\hline $\mathrm{Cr}_{2} \mathrm{O}_{3}$ & 0.02 & $b d l$ & $b d l$ & 0.01 & 0.03 & $b d l$ & $b d l$ & 0.02 & $b d l$ & $b d l$ \\
\hline $\mathrm{FeO}$ & 9.05 & 8.97 & 8.93 & 9.10 & 8.82 & 10.22 & 10.38 & 10.08 & 10.06 & 10.33 \\
\hline MnO & 0.13 & 0.16 & 0.12 & 0.15 & 0.13 & 0.17 & 0.17 & 0.15 & 0.15 & 0.15 \\
\hline MgO & 50.54 & 49.92 & 50.83 & 50.04 & 50.51 & 49.24 & 49.13 & 49.64 & 49.54 & 49.71 \\
\hline $\mathrm{CaO}$ & 0.03 & 0.04 & 0.04 & 0.05 & 0.03 & 0.02 & 0.01 & $b d l$ & 0.02 & 0.01 \\
\hline $\mathrm{Na}_{2} \mathrm{O}$ & $b d l$ & $b d l$ & 0.03 & $b d l$ & 0.02 & 0.03 & 0.01 & 0.02 & $b d l$ & $b d l$ \\
\hline $\mathrm{NiO}$ & 0.44 & 0.43 & 0.42 & 0.41 & 0.42 & 0.50 & 0.49 & 0.45 & 0.45 & 0.47 \\
\hline Total & 100.83 & 100.31 & 101.36 & 100.47 & 101.18 & 100.75 & 100.84 & 100.77 & 100.77 & 100.97 \\
\hline \multicolumn{11}{|c|}{ Formula based on 4 oxygens } \\
\hline Si (apfu) & 0.99 & 1.00 & 0.99 & 0.99 & 1.00 & 0.99 & 0.99 & 0.99 & 0.99 & 0.98 \\
\hline $\mathrm{Ti}$ & - & - & - & - & - & - & - & - & - & 0.00 \\
\hline Al & - & - & - & 0.00 & 0.00 & 0.00 & - & - & 0.00 & 0.00 \\
\hline $\mathbf{V}$ & 0.00 & 0.00 & - & - & - & 0.00 & 0.00 & 0.00 & - & 0.00 \\
\hline $\mathrm{Cr}$ & 0.00 & - & - & 0.00 & 0.00 & - & - & 0.00 & - & - \\
\hline $\mathrm{Fe}^{2+}$ & 0.18 & 0.18 & 0.18 & 0.19 & 0.18 & 0.21 & 0.21 & 0.21 & 0.21 & 0.21 \\
\hline Mn & 0.00 & 0.00 & 0.00 & 0.00 & 0.00 & 0.00 & 0.00 & 0.00 & 0.00 & 0.00 \\
\hline Mg & 1.83 & 1.82 & 1.83 & 1.82 & 1.82 & 1.79 & 1.79 & 1.81 & 1.80 & 1.81 \\
\hline $\mathrm{Ca}$ & 0.00 & 0.00 & 0.00 & 0.00 & 0.00 & 0.00 & 0.00 & - & 0.00 & 0.00 \\
\hline $\mathrm{Na}$ & - & - & 0.00 & - & 0.00 & 0.00 & 0.00 & 0.00 & - & - \\
\hline $\mathbf{N i}$ & 0.01 & 0.01 & 0.01 & 0.01 & 0.01 & 0.01 & 0.01 & 0.01 & 0.01 & 0.01 \\
\hline Fo & 90.87 & 90.84 & 91.03 & 90.74 & 90.78 & 89.57 & 89.41 & 89.78 & 89.78 & 89.56 \\
\hline
\end{tabular}

*bdl-below detection limit

grains; Figures 4C to 4N). The PGM grains have sizes ranging from 5 to $25 \mu \mathrm{m}$ and they occur as single inclusions or as polyphasic inclusions. The main PGM observed in the samples is euhedral to subhedral laurite (Figure 4C and 4D). Laurite grains may display growth bands (Figure 4D. They may contain acicular oriented Ir exsolutions along crystallographic planes (Figure 4E) or show oscillatory zoning (Figure 4F). Locally, laurite grains contain nanometric mineral inclusions of $\mathrm{Cu}-\mathrm{Fe}$ sulfides and Pt-Fe and Ir alloys (Figures $4 \mathrm{G}$ to $4 \mathrm{H}$ and $4 \mathrm{M}$ to $4 \mathrm{~N}$ ). Some of the laurite grains exhibit corroded surfaces with rugged textures (Figures $4 \mathrm{~L}$ to $4 \mathrm{~N}$ ). Another type of PGM observed in the chromitite samples consists of grains Ir-Rh-Pt(-Cu-Co-Ni) composition. This type of PGM occurs systematically associated with laurite grains as overgrowths on the surfaces of the crystals (Figures $4 \mathrm{G}$ to $4 \mathrm{~K}$ ). They show a wide range of morphologies and textures, such as micrometric clustered irregular grains (Figures 4G to $4 \mathrm{H}$ ), nanometric aggregates (Figure $4 \mathrm{~K}$ ) and grain-forming fibers with slightly radial disposition (Figure 4J). 
Table 2. Representative electron microprobe analyses of orthopyroxene from harzburgites $(\mathrm{Hz})$ and orthopyroxenites (Opyr) from the Havana-Matanzas Ophiolite. $\mathrm{Mg} \#=\mathrm{Mg} /\left(\mathrm{Mg}+\mathrm{Fe}^{2+}\right)$.

\begin{tabular}{|c|c|c|c|c|c|c|c|c|c|c|}
\hline Rock type & $\mathrm{Hz}$ & $\mathrm{Hz}$ & $\mathrm{Hz}$ & $\mathrm{Hz}$ & $\mathrm{Hz}$ & Opyr & Opyr & Opyr & Opyr & Opyr \\
\hline $\mathrm{SiO}_{2}($ wt. $\%)$ & 55.69 & 55.70 & 56.00 & 55.68 & 55.53 & 56.16 & 56.60 & 57.02 & 56.76 & 56.40 \\
\hline $\mathrm{TiO}_{2}$ & 0.02 & 0.04 & 0.02 & 0.03 & 0.04 & 0.02 & 0.03 & 0.05 & 0.06 & 0.04 \\
\hline $\mathbf{A l}_{2} \mathbf{O}_{3}$ & 2.60 & 2.70 & 2.47 & 2.59 & 2.55 & 1.08 & 1.06 & 1.05 & 1.03 & 1.12 \\
\hline $\mathrm{V}_{2} \mathrm{O}_{3}$ & 0.05 & 0.02 & 0.01 & 0.01 & $b d l$ & $b d l$ & 0.02 & $b d l$ & $b d l$ & $b d l$ \\
\hline $\mathrm{Cr}_{2} \mathrm{O}_{3}$ & 0.80 & 0.75 & 0.69 & 0.82 & 0.75 & 0.38 & 0.33 & 0.36 & 0.37 & 0.40 \\
\hline $\mathrm{FeO}$ & 6.00 & 5.86 & 5.72 & 5.91 & 5.98 & 6.87 & 6.94 & 6.84 & 6.89 & 6.86 \\
\hline $\mathrm{MnO}$ & 0.14 & 0.16 & 0.16 & 0.12 & 0.16 & 0.15 & 0.18 & 0.17 & 0.17 & 0.21 \\
\hline MgO & 34.36 & 33.03 & 34.04 & 34.27 & 33.83 & 34.19 & 34.98 & 34.51 & 34.85 & 34.64 \\
\hline $\mathrm{CaO}$ & 0.97 & 2.20 & 1.66 & 1.25 & 0.97 & 1.04 & 0.95 & 0.91 & 0.61 & 1.08 \\
\hline $\mathrm{Na}_{2} \mathrm{O}$ & 0.01 & $b d l$ & $b d l$ & $b d l$ & 0.03 & 0.01 & 0.03 & $b d l$ & $b d l$ & 0.01 \\
\hline $\mathrm{NiO}$ & 0.13 & 0.12 & 0.08 & 0.09 & 0.10 & 0.12 & 0.07 & 0.06 & 0.12 & 0.09 \\
\hline Total & 100.77 & 100.57 & 100.85 & 100.76 & 99.94 & 100.02 & 101.20 & 100.98 & 100.85 & 100.83 \\
\hline \multicolumn{11}{|c|}{ Formula based on 6 oxygens } \\
\hline Si (apfu) & 1.92 & 1.92 & 1.92 & 1.91 & 1.92 & 1.95 & 1.94 & 1.96 & 1.95 & 1.94 \\
\hline $\mathbf{T i}$ & 0.00 & 0.00 & 0.00 & 0.00 & 0.00 & 0.00 & 0.00 & 0.00 & 0.00 & 0.00 \\
\hline Al & 0.11 & 0.11 & 0.10 & 0.11 & 0.10 & 0.04 & 0.04 & 0.04 & 0.04 & 0.05 \\
\hline $\mathbf{V}$ & 0.00 & 0.00 & 0.00 & 0.00 & - & - & 0.00 & - & - & - \\
\hline $\mathbf{C r}$ & 0.02 & 0.02 & 0.02 & 0.02 & 0.02 & 0.01 & 0.01 & 0.01 & 0.01 & 0.01 \\
\hline $\mathrm{Fe}^{2+}$ & 0.17 & 0.17 & 0.16 & 0.17 & 0.17 & 0.20 & 0.20 & 0.20 & 0.20 & 0.20 \\
\hline Mn & 0.00 & 0.01 & 0.01 & 0.00 & 0.01 & 0.00 & 0.01 & 0.01 & 0.01 & 0.01 \\
\hline Mg & 1.76 & 1.70 & 1.74 & 1.76 & 1.75 & 1.77 & 1.79 & 1.77 & 1.79 & 1.78 \\
\hline $\mathrm{Ca}$ & 0.04 & 0.08 & 0.06 & 0.05 & 0.04 & 0.04 & 0.04 & 0.03 & 0.02 & 0.04 \\
\hline $\mathrm{Na}$ & 0.00 & - & - & - & 0.00 & 0.00 & 0.00 & - & - & 0.00 \\
\hline $\mathbf{N i}$ & 0.00 & 0.00 & 0.00 & 0.00 & 0.00 & 0.00 & 0.00 & 0.00 & 0.00 & 0.00 \\
\hline Mg\# & 0.92 & 0.91 & 0.92 & 0.89 & 0.91 & 0.90 & 0.90 & 0.90 & 0.90 & 0.90 \\
\hline En & 89.38 & 87.33 & 88.86 & 88.95 & 89.32 & 88.09 & 88.21 & 88.52 & 89.06 & 88.15 \\
\hline $\mathrm{Fe}$ & 8.63 & 8.73 & 8.17 & 8.59 & 8.68 & 9.95 & 9.86 & 10.00 & 9.95 & 9.90 \\
\hline Wo & 1.99 & 3.94 & 2.97 & 2.46 & 2.00 & 1.95 & 1.93 & 1.48 & 0.99 & 1.94 \\
\hline
\end{tabular}

\section{Mineral chemistry}

\subsection{OLIVINE}

Olivine from the harzburgite shows a typical mantle composition with forsterite content $(\mathrm{Fo}=100$ $\mathrm{x} \mathrm{Mg} /\left[\mathrm{Mg}+\mathrm{Fe}^{2+}\right]$, atomic ratio) between 90.7 and 91.1, $\mathrm{NiO}$ contents between 0.42 and 0.44 wt. $\%$, and $\mathrm{MnO}$ between 0.12 and 0.16 wt.\% (Table 1). Olivine from the orthopyroxenite bands shows slightly lower Fo values (89.1-89.8) and similar $\mathrm{NiO}$ and $\mathrm{MnO}$ contents $(0.43-0.50$ wt. $\%$ and $0.11-0.18$ wt. $\%$, respectively) to olivine from harzburgite (Table 1).

\subsection{ORTHOPYROXENE}

Orthopyroxene in harzburgite is very rich in $\mathrm{Mg}$, with $\mathrm{Mg} \#\left(\mathrm{Mg} /\left[\mathrm{Mg}+\mathrm{Fe}^{2+}\right]\right.$, atomic ratio) of 0.91 , corresponding to enstatite $\left(\mathrm{En}_{87.17-89.52} \mathrm{Fe}_{8.17-10.16} \mathrm{Wo}_{0.99-4.00}\right)$. It contains 2.4-2.6 wt. $\% \quad \mathrm{Al}_{2} \mathrm{O}_{3}, 0.7-0.8$ wt. $\%$ $\mathrm{Cr}_{2} \mathrm{O}_{3}$, and $0.01-0.04$ wt. $\% \mathrm{TiO}_{2}$ (Table 2). Orthopyroxene from the orthopyroxenite bands is slightly less magnesian $(\mathrm{Mg} \#=0.89-0.90)$, but still corresponds to enstatite in composition $\left(\mathrm{En}_{\text {89.69 90.03 }} \mathrm{Fe}_{9.97-}\right.$ $\left.{ }_{10.31} \mathrm{Wo}_{1.12-3.14}\right)$. Compared to orthopyroxene from the harzburgites, it has lower $\mathrm{Al}_{2} \mathrm{O}_{3}$ and $\mathrm{Cr}_{2} \mathrm{O}_{3}$ contents (0.9-1.2 wt. $\%$ and $0.3-0.5$ wt. $\%$, respectively) but similar $\mathrm{TiO}_{2}$ content $(0.02-0.06$ wt. \%) (Table 2). 
Table 3. Representative electron microprobe analyses of chromite from harzburgites $(\mathrm{Hz})$, orthopyroxenites (Opyr) and chromitite veins (VChr) from the Havana-Matanzas Ophiolite. $\mathrm{Cr} \#=\mathrm{Cr} /(\mathrm{Cr}+\mathrm{Al}) ; \mathrm{Mg} \#=\mathrm{Mg} /\left(\mathrm{Mg}+\mathrm{Fe}^{2+}\right)$.

\begin{tabular}{|c|c|c|c|c|c|c|c|c|c|c|}
\hline Rock type & $\mathrm{Hz}$ & $\mathrm{Hz}$ & $\mathrm{Hz}$ & $\mathrm{Hz}$ & $\mathrm{Hz}$ & $\mathrm{Hz}$ & $\mathrm{Hz}$ & $\mathrm{Hz}$ & Opyr & Opyr \\
\hline $\mathrm{SiO}_{2}$ (wt. \%) & $b d l$ & 0.00 & 0.02 & 0.03 & 0.05 & 0.01 & 0.03 & 0.04 & 0.01 & 0.00 \\
\hline $\mathrm{TiO}_{2}$ & 0.03 & 0.03 & 0.07 & 0.05 & 0.05 & 0.05 & 0.05 & 0.03 & 0.19 & 0.19 \\
\hline $\mathrm{V}_{2} \mathrm{O}_{5}$ & 0.22 & 0.18 & 0.20 & 0.19 & 0.22 & 0.24 & 0.22 & 0.18 & 0.25 & 0.26 \\
\hline $\mathrm{Al}_{2} \mathrm{O}_{3}$ & 30.43 & 32.03 & 27.99 & 28.16 & 27.14 & 28.22 & 30.27 & 30.27 & 15.52 & 15.67 \\
\hline $\mathrm{Cr}_{2} \mathrm{O}_{3}$ & 35.20 & 34.83 & 38.57 & 37.73 & 40.16 & 38.74 & 37.81 & 37.62 & 45.81 & 46.16 \\
\hline $\mathrm{FeO}(\mathrm{d})$ & 12.81 & 13.70 & 12.54 & 12.70 & 13.95 & 13.27 & 12.68 & 12.59 & 20.51 & 20.62 \\
\hline $\mathrm{Fe}_{2} \mathrm{O}_{3}(\&)$ & 4.85 & 3.08 & 5.24 & 6.07 & 4.15 & 4.63 & 4.02 & 4.19 & 8.05 & 7.79 \\
\hline MgO & 16.07 & 15.71 & 15.56 & 15.46 & 14.54 & 15.12 & 15.86 & 15.85 & 8.85 & 8.90 \\
\hline $\mathrm{MnO}$ & 0.20 & 0.24 & 0.25 & 0.29 & 0.29 & 0.28 & 0.24 & 0.26 & 0.43 & 0.38 \\
\hline NiO & 0.11 & 0.15 & 0.13 & 0.15 & 0.14 & 0.12 & 0.14 & 0.14 & 0.09 & 0.11 \\
\hline Total & 99.92 & 99.95 & 100.05 & 100.21 & 100.27 & 100.21 & 100.92 & 100.58 & 98.91 & 99.31 \\
\hline \multicolumn{11}{|c|}{ Formula based on 32 oxygens } \\
\hline Ti (apfu) & 0.00 & 0.00 & 0.00 & 0.00 & 0.00 & 0.00 & 0.00 & 0.00 & 0.01 & 0.01 \\
\hline $\mathbf{A l}$ & 1.10 & 1.12 & 0.97 & 0.98 & 0.95 & 0.98 & 1.04 & 1.04 & 0.60 & 0.60 \\
\hline $\mathbf{V}$ & 0.00 & 0.00 & 0.00 & 0.00 & 0.00 & 0.00 & 0.00 & 0.00 & 0.00 & 0.00 \\
\hline $\mathrm{Cr}$ & 0.79 & 0.78 & 0.49 & 0.88 & 0.95 & 0.91 & 0.87 & 0.86 & 1.19 & 1.19 \\
\hline $\mathrm{Fe}^{2+}(\mathcal{\&})$ & 0.30 & 0.33 & 0.31 & 0.31 & 0.35 & 0.33 & 0.31 & 0.31 & 0.56 & 0.56 \\
\hline $\mathrm{Fe}^{3+}(\&)$ & 0.10 & 0.07 & 0.12 & 0.14 & 0.09 & 0.10 & 0.09 & 0.09 & 0.20 & 0.19 \\
\hline Mg & 0.69 & 0.67 & 0.69 & 0.68 & 0.65 & 0.67 & 0.69 & 0.69 & 0.43 & 0.43 \\
\hline Mn & 0.01 & 0.01 & 0.01 & 0.01 & 0.01 & 0.01 & 0.01 & 0.01 & 0.01 & 0.01 \\
\hline $\mathbf{N i}$ & 0.01 & 0.00 & 0.00 & 0.00 & 0.00 & 0.00 & 0.00 & 0.00 & 0.00 & 0.00 \\
\hline Cr\# & 0.42 & 0.41 & 0.48 & 0.47 & 0.50 & 0.48 & 0.46 & 0.45 & 0.66 & 0.66 \\
\hline Mg\# & 0.69 & 0.67 & 0.68 & 0.68 & 0.65 & 0.67 & 0.69 & 0.69 & 0.43 & 0.43 \\
\hline \multicolumn{11}{|c|}{$* b d l=$ below detection limit; $\&=$ estimated by stoichiometry } \\
\hline Rock type & Opyr & Opyr & Opyr & Opyr & Opyr & VChr & VChr & VChr & VChr & VChr \\
\hline $\mathrm{SiO}_{2}($ wt. \%) & $b d l$ & 0.01 & 0.04 & 0.05 & 0.01 & 0.04 & 0.03 & 0.06 & 0.07 & 0.03 \\
\hline $\mathrm{TiO}_{2}$ & 0.18 & 0.22 & 0.23 & 0.19 & 0.23 & 0.25 & 0.26 & 0.22 & 0.24 & 0.24 \\
\hline $\mathrm{V}_{2} \mathrm{O}_{5}$ & 0.27 & 0.22 & 0.29 & 0.28 & 0.25 & 0.22 & 0.24 & 0.22 & 0.21 & 0.20 \\
\hline $\mathrm{Al}_{2} \mathrm{O}_{3}$ & 14.79 & 14.96 & 15.77 & 15.26 & 15.70 & 13.38 & 13.22 & 13.14 & 13.03 & 13.31 \\
\hline $\mathrm{Cr}_{2} \mathrm{O}_{3}$ & 46.33 & 48.86 & 47.98 & 48.04 & 47.88 & 51.22 & 51.34 & 51.73 & 51.79 & 51.92 \\
\hline $\mathrm{FeO}(\&)$ & 20.01 & 17.80 & 18.03 & 18.30 & 18.35 & 14.47 & 14.69 & 12.61 & 14.45 & 15.04 \\
\hline $\mathrm{Fe}_{2} \mathrm{O}_{3}(\&)$ & 8.05 & 6.87 & 7.23 & 6.88 & 6.52 & 7.08 & 6.92 & 8.84 & 7.29 & 6.23 \\
\hline MgO & 9.07 & 10.68 & 10.78 & 10.29 & 10.38 & 12.68 & 12.47 & 14.19 & 12.75 & 12.26 \\
\hline $\mathrm{MnO}$ & 0.42 & 0.39 & 0.38 & 0.39 & 0.33 & 0.29 & 0.34 & 0.28 & 0.29 & 0.34 \\
\hline $\mathrm{NiO}$ & 0.09 & 0.12 & 0.13 & 0.15 & 0.12 & 0.14 & 0.15 & 0.13 & 0.13 & 0.13 \\
\hline Total & 98.69 & 99.45 & 100.14 & 99.13 & 99.12 & 99.05 & 98.97 & 100.53 & 99.52 & 99.06 \\
\hline \multicolumn{11}{|c|}{ Formula based on 32 oxygens } \\
\hline Ti (apfu) & 0.00 & 0.01 & 0.01 & 0.00 & 0.01 & 0.01 & 0.01 & 0.01 & 0.01 & 0.01 \\
\hline Al & 0.57 & 0.57 & 0.59 & 0.58 & 0.60 & 0.51 & 0.50 & 0.49 & 0.49 & 0.51 \\
\hline $\mathbf{V}$ & 0.00 & 0.00 & 0.00 & 0.00 & 0.00 & 0.00 & 0.00 & 0.00 & 0.00 & 0.00 \\
\hline $\mathbf{C r}$ & 1.20 & 1.25 & 1.21 & 1.23 & 1.22 & 1.30 & 1.31 & 1.29 & 1.31 & 1.32 \\
\hline $\mathrm{Fe}^{2+}(\&)$ & 0.55 & 0.48 & 0.48 & 0.50 & 0.50 & 0.39 & 0.40 & 0.33 & 0.39 & 0.41 \\
\hline $\mathrm{Fe}^{3+}(\&)$ & 0.21 & 0.17 & 0.17 & 0.17 & 0.16 & 0.17 & 0.17 & 0.21 & 0.18 & 0.15 \\
\hline $\mathbf{M g}$ & 0.44 & 0.51 & 0.51 & 0.50 & 0.50 & 0.61 & 0.60 & 0.67 & 0.61 & 0.59 \\
\hline Mn & 0.01 & 0.01 & 0.01 & 0.01 & 0.01 & 0.01 & 0.01 & 0.01 & 0.01 & 0.01 \\
\hline $\mathbf{N i}$ & 0.00 & 0.00 & 0.00 & 0.00 & 0.00 & 0.00 & 0.00 & 0.00 & 0.00 & 0.00 \\
\hline Cr\# & 0.68 & 0.69 & 0.67 & 0.68 & 0.67 & 0.72 & 0.72 & 0.73 & 0.73 & 0.72 \\
\hline Mg\# & 0.45 & 0.52 & 0.52 & 0.50 & 0.50 & 0.61 & 0.60 & 0.67 & 0.61 & 0.59 \\
\hline
\end{tabular}




\subsection{CHROMITE}

Accessory chromite from harzburgite is high- $\mathrm{Al}$ in composition $(\mathrm{Cr} \#=0.39-0.50$; Figure $5 \mathrm{~A})$, with 0.65-0.71 Mg\# values, $\mathrm{Al}_{2} \mathrm{O}_{3}$ contents between 27 and 39 wt. $\%, \mathrm{Fe}_{2} \mathrm{O}_{3}$ contents between 2.71 and 6.39 wt. $\%$, and $\mathrm{TiO}_{2}$ contents between 0.02 and 0.07 wt. \% (Figures 5B to 5D; Table 3). Conversely, chromite from the orthopyroxenite dykes is high-Cr in composition $(\mathrm{Cr} \#=0.62-0.69$; Figure $5 \mathrm{~A})$, with lower $\mathrm{Mg} \#$ values (0.39-0.52), lower $\mathrm{Al}_{2} \mathrm{O}_{3}$ contents (14-16 wt.\%) and higher $\mathrm{Fe}_{2} \mathrm{O}_{3}$ and $\mathrm{TiO}_{2}$ contents than the chromite from harzburgite (6.52-9.67 wt. $\%$ and $0.16-0.23$ wt. $\%$, respectively; Figures 5B to 5D; Table 3). Chromite from the chromitite veins shows similar composition to accessory chromite from the orthopyroxenite veins (Table 3): high-Cr chromite $(\mathrm{Cr} \#=0.72-0.73)$ and $0.59-0.67 \mathrm{Mg} \#$ values, with 11.67-13.62 wt. $\% \mathrm{Al}_{2} \mathrm{O}_{3}, 6.23-8.84$ wt. $\% \mathrm{Fe}_{2} \mathrm{O}_{3}$ and $0.17-0.26$ wt. $\% \mathrm{TiO}_{2}$ contents (Figures 5A to 5D). The chromite composition of the chromitite veins is homogeneous across the veins, with no chemical variations at the contact with the host orthopyroxenite (Figure 5E).

\subsection{PLATINUM GROUP MINERALS (PGM)}

Laurite shows varied composition $\left[\left(\mathrm{Ru}_{0.64-0.78} \mathrm{Os}_{0.16-}\right.\right.$ $\left.\left.{ }_{0.19} \mathrm{Ir}_{0.06-0.12} \mathrm{Fe}_{0.01-0.02}\right) \mathrm{S}_{1.94-2.06}\right]$ (Figure 6; Table 4), classifying as Os-rich laurites. Laurite analyses also show $\mathrm{Ni}(0.13-0.44$ wt.\%), $\mathrm{Cu}$ (up to 0.09 wt. $\%$ ) and Co (0.01-0.11 wt. \%) that, together with the amount of $\mathrm{Fe}$, suggest contamination of the analyses by nanometric Ni-Fe-Cu sulfide inclusions, which can be locally observed within the laurite grains (Figure $4 \mathrm{H})$.

\section{Discussion}

\subsection{PETROGENESIS OF ULTRAMAFIC ROGKS FROM HAVANA-MATANZAS OPHIOLITE}

The composition of minerals forming the harzburgites and the orthopyroxenite bands from the Havana-Matanzas Ophiolite suggests that both types of rock have different origin and petrogenetic evolution. Olivine and orthopyroxene from harzburgite show compositions typical of residual rocks derived from partial melting of primitive mantle (e.g., Hirauchi et al., 2008; Pagé et al., 2008). The degree of partial melting $\mathrm{F}(\%)$ of these rocks can be inferred from the Cr\# of chromite, using the following equation: $\mathrm{F}=10 \times \ln (\mathrm{Cr} \#)+24$ (Hellebrand et al., 2001). According to this equation, the sampled Havana-Matanzas Ophiolite harzburgites experienced 15 to $17 \%$ partial melting. The composition of accessory chromite corresponds to chromite derived from abyssal peridotites of mid-ocean ridge or back-arc settings (Figures 5B and 5D). This suggests that the harzburgites are residual peridotites resulting from melt extraction in a slow to ultra-slow spreading mid-ocean ridge setting or back-arc basin (e.g., Hellebrand et al., 2001; Hirauchi et al., 2008). On the other hand, the replacement of orthopyroxene by olivine reflects the incongruent dissolution of orthopyroxene and consequent crystallization of olivine occurring in the mass transfer reaction between peridotite and a fractionating basalt (Kelemen, 1990). This is typical in subduction-related magmatic arcs, where tholeiitic primary liquids pass slowly upwards through high-temperature wall rock in the upper mantle (Kelemen, 1990).

Conversely, chromite from the orthopyroxenite bands shows compositions which are typical of chromite from boninite-like magmas (Figure 5C), providing evidence for the hypothesis that the orthopyroxenites formed from boninitic magmas in a fore-arc setting, probably during subduction initiation stages of a nascent intra-oceanic arc (Pagé et al., 2008; Whattam and Stern, 2011). The orthopyroxenite bands represent a reaction product between the upward migrating melt and the host peridotite in the upper mantle. The liquid from which the orthopyroxenite formed represents a relatively $\mathrm{Si}$-rich $\mathrm{Mg}$-rich andesite melt (boninitic-like) that migrated through the mantle harzburgite (e.g., Berly et al., 2006; Grant et al., 2016; Wang et al., 2016) and that became Si-enriched by reactive dissolution of mantle pyroxene. Therefore, the orthopyroxenite bands from Havana-Matanzas Ophiolite are inter- 

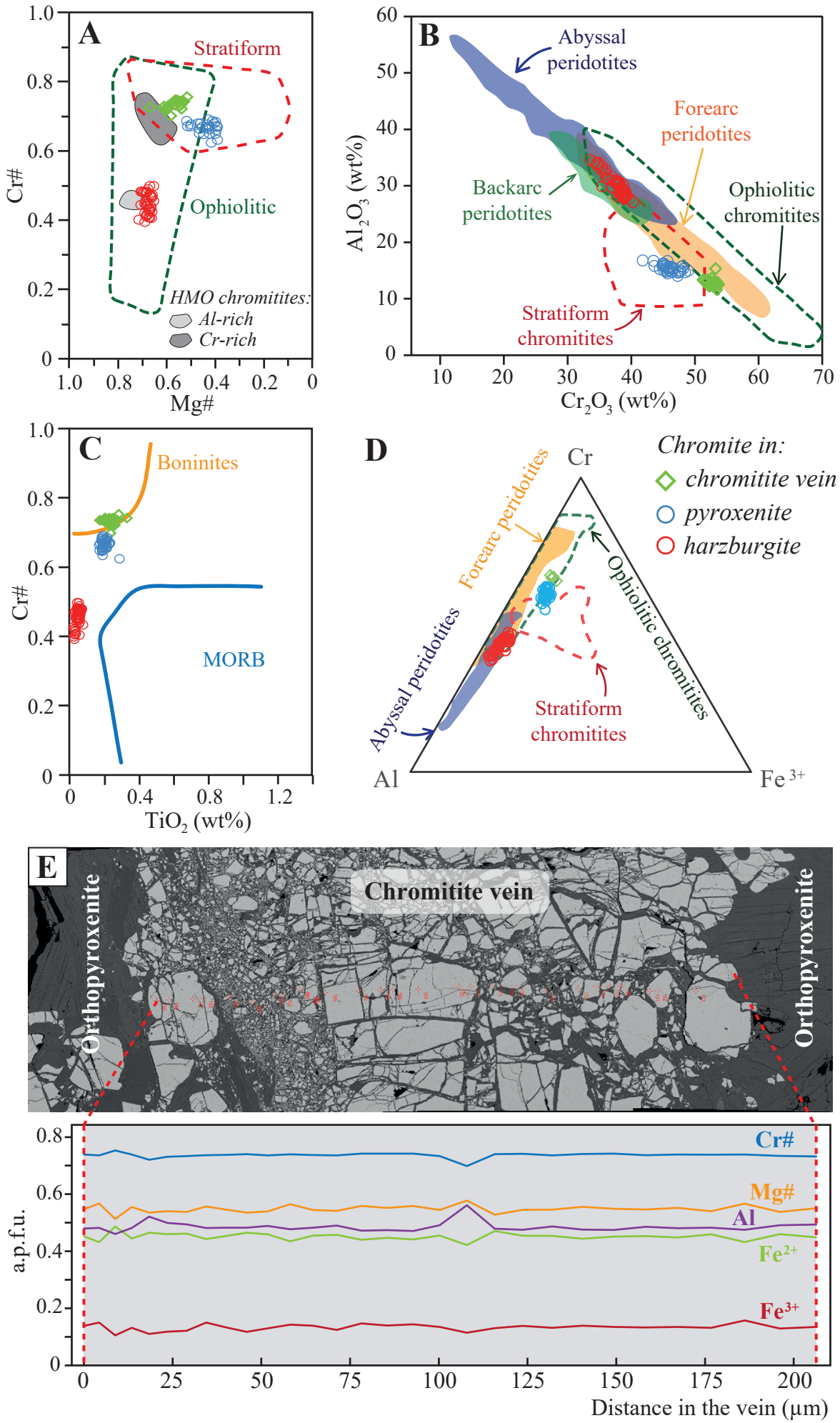

Figure 5 Chemical composition of chromite from the studied samples. A: $\mathrm{Cr} \#[\mathrm{Cr} /(\mathrm{Cr}+\mathrm{Al})] \mathrm{vs}$. Mg\# $\left[\mathrm{Mg} /\left(\mathrm{Mg}+\mathrm{Fe}^{2+}\right)\right] \mathrm{diagram}$. Compilation of the composition fields from Leblanc and Nicolas (1992). Al-rich and Cr-rich chromitites fields from Llanes-Castro et al. (2015). B: $\mathrm{Al}_{2} \mathrm{O}_{3}$ vs. $\mathrm{Cr}_{2} \mathrm{O}_{3}$ diagram. Ophiolitic and stratiform chromitites fields from Bonavia et al. (1993). C: $\mathrm{Cr} \#$ vs. $\mathrm{TiO}_{2}$ diagram. Composition fields from Arai (1992). D: Cr-Al-Fe ${ }^{3+}$ atomic ratios diagram. Ophiolitic and stratiform chromitite fields are from Ferrario and Garuti (1987) and Arai et al. (2004). Abyssal and forearc peridotite fields are from Ishii et al. (1992) and Dick and Bullen (1984), respectively. $\mathrm{E}$ : Composition profile of chromite across the chromitite vein. HMO = Havana-Matanzas Ophiolite. 
Table 4. Representative electron microprobe analyses of the PGM from chromitite veins of the Havana-Matanzas Ophiolite.

\begin{tabular}{|c|c|c|c|c|c|c|c|c|c|c|c|c|}
\hline Mineral & $\begin{array}{l}\text { Os-rich } \\
\text { laurite }\end{array}$ & $\begin{array}{l}\text { Os-rich } \\
\text { laurite }\end{array}$ & $\begin{array}{l}\text { Os-rich } \\
\text { laurite }\end{array}$ & $\begin{array}{l}\text { Os-rich } \\
\text { laurite }\end{array}$ & $\begin{array}{l}\text { Os-rich } \\
\text { laurite }\end{array}$ & $\begin{array}{l}\text { Os-rich } \\
\text { laurite }\end{array}$ & $\begin{array}{l}\text { Os-rich } \\
\text { laurite }\end{array}$ & $\begin{array}{l}\text { Os-rich } \\
\text { laurite }\end{array}$ & $\begin{array}{l}\text { Os-rich } \\
\text { laurite }\end{array}$ & $\begin{array}{l}\text { Os-rich } \\
\text { laurite }\end{array}$ & $\begin{array}{l}\text { Os-rich } \\
\text { laurite }\end{array}$ & $\begin{array}{l}\text { Os-rich } \\
\text { laurite }\end{array}$ \\
\hline Os (wt.\%) & 16.57 & 17.54 & 16.09 & 15.94 & 16.84 & 16.35 & 18.97 & 17.23 & 18.01 & 15.45 & 15.97 & 15.67 \\
\hline Ir & 8.60 & 9.20 & 8.30 & 9.21 & 6.86 & 10.08 & 6.60 & 7.27 & 12.07 & 9.52 & 10.06 & 10.12 \\
\hline $\mathbf{R u}$ & 35.31 & 33.46 & 37.18 & 38.17 & 40.59 & 35.09 & 39.47 & 39.06 & 34.38 & 36.04 & 36.28 & 36.34 \\
\hline $\mathbf{P t}$ & 0.23 & 0.41 & $b d l$ & 0.13 & $b d l$ & $b d l$ & $b d l$ & $b d l$ & $b d l$ & $b d l$ & $b d l$ & $b d l$ \\
\hline $\mathbf{P d}$ & 0.09 & 0.04 & 0.04 & 0.02 & 0.17 & 0.61 & $b d l$ & $b d l$ & 0.11 & 0.16 & 0.30 & 0.22 \\
\hline $\mathbf{R h}$ & 0.52 & 0.42 & 0.73 & 0.71 & $b d l$ & 0.59 & 0.13 & 0.46 & 0.41 & 1.04 & 1.04 & 1.01 \\
\hline $\mathrm{Fe}$ & 0.32 & 0.46 & 0.56 & 0.52 & 0.34 & 0.67 & 0.30 & 0.45 & 0.26 & 0.33 & 0.37 & 0.30 \\
\hline $\mathbf{N i}$ & 0.27 & 0.29 & 0.38 & 0.30 & 0.23 & 0.44 & 0.22 & 0.31 & 0.13 & 0.27 & 0.25 & 0.19 \\
\hline $\mathrm{Cu}$ & 0.08 & 0.05 & 0.07 & 0.01 & 0.06 & 0.02 & 0.03 & $b d l$ & 0.02 & 0.08 & 0.09 & $b d l$ \\
\hline Co & 0.11 & 0.02 & 0.06 & 0.10 & 0.07 & 0.06 & 0.01 & 0.04 & 0.02 & 0.03 & 0.05 & 0.04 \\
\hline $\mathbf{S}$ & 34.26 & 34.27 & 35.27 & 31.12 & 32.12 & 31.66 & 33.70 & 33.90 & 32.76 & 33.10 & 33.81 & 32.87 \\
\hline Total & 96.36 & 96.15 & 98.68 & 96.24 & 97.28 & 95.56 & 99.44 & 98.72 & 98.17 & 96.01 & 98.23 & 96.77 \\
\hline Os (apfu) & 0.17 & 0.18 & 0.16 & 0.17 & 0.17 & 0.17 & 0.19 & 0.17 & 0.19 & 0.16 & 0.16 & 0.16 \\
\hline Ir & 0.09 & 0.09 & 0.08 & 0.10 & 0.07 & 0.11 & 0.06 & 0.07 & 0.12 & 0.10 & 0.10 & 0.10 \\
\hline $\mathbf{R u}$ & 0.67 & 0.64 & 0.68 & 0.75 & 0.78 & 0.70 & 0.74 & 0.73 & 0.67 & 0.69 & 0.69 & 0.70 \\
\hline $\mathbf{P t}$ & 0.00 & 0.00 & - & 0.00 & - & - & - & - & - & - & - & - \\
\hline $\mathbf{R h}$ & 0.01 & 0.01 & 0.01 & 0.01 & 0.00 & 0.01 & 0.00 & 0.01 & 0.01 & 0.02 & 0.02 & 0.02 \\
\hline $\mathrm{Fe}^{2+}$ & 0.01 & 0.02 & 0.02 & 0.02 & 0.01 & 0.02 & 0.01 & 0.02 & 0.01 & 0.01 & 0.01 & 0.01 \\
\hline $\mathrm{Ni}$ & 0.01 & 0.01 & 0.01 & 0.01 & 0.01 & 0.01 & 0.01 & 0.01 & 0.00 & 0.01 & 0.01 & 0.01 \\
\hline $\mathbf{S}$ & 2.05 & 2.06 & 2.04 & 1.94 & 1.95 & 1.98 & 1.99 & 1.99 & 2.00 & 2.01 & 2.01 & 2.00 \\
\hline
\end{tabular}

*bdl-below detection limit

preted as the "footprint" of boninitic-affinity melt circulation in the mantle, which gives place to dykes and lavas in the upper crustal parts of the ophiolitic sequence (e.g., Varfalvy et al., 1997). Similar parental melts were inferred for orthopyroxenites in the Speik Complex (Eastern Alps, Austria), which are interlayered with abundant pods and layers of chromitites (Melcher and Meisel, 2004). Using the composition of orthopyroxene, olivine, and chromite from the orthopyroxenite bands, we applied several geothermometers (Brey and Köhler, 1990; Köhler and Brey, 1990) and obtained equilibrium temperatures for the orthopyroxenite samples between 700 and $1100^{\circ} \mathrm{C}$, which we interpret as post-interaction cooling temperatures.

\subsection{PETROGENESIS OF THE GHROMITITE VEINS AND THE PGE MINERALIZATIONS}

\subsubsection{CHROMITITE VEINS HOSTED IN THE ORTHOPYROXENITE BANDS}

Major element composition of chromite from the chromitite veins is similar to that of chromite formed from boninitic magmas (Figure 5C) (e.g., González-Jiménez et al., 2014, and references therein). The composition of the chromitite parental melt can be inferred from the composition of their crystallized chromite grains using the following equations (Zaccarini et al., 2011):

$$
\begin{gathered}
\mathrm{Al}_{2} \mathrm{O}_{3 \text { melt }}=4.1385 \times \ln \left(\mathrm{Al}_{2} \mathrm{O}_{3 \text { chromite }}\right)+2.2828 \\
\mathrm{TiO}_{2 \text { melt }}=0.708 \times \ln \left(\mathrm{TiO}_{2 \text { chromite }}\right)+1.636
\end{gathered}
$$

$\ln (\mathrm{FeO} / \mathrm{MgO})_{\text {chromite }}=0.47-1.07 \mathrm{Al} \#_{\text {chromite }}+$ $0.64 \mathrm{Fe} \#_{\text {chromite }}+\ln (\mathrm{FeO} / \mathrm{MgO})_{\text {melt }}$

with $\mathrm{Al} \#_{\text {chromite }}=\mathrm{Al} /\left(\mathrm{Cr}+\mathrm{Al}+\mathrm{Fe}^{3+}\right)$ atomic ratio, and $\mathrm{Fe} \#_{\text {chromite }}=\mathrm{Fe}^{3+} /\left(\mathrm{Cr}+\mathrm{Al}+\mathrm{Fe}^{3+}\right)$ atomic ratio. The calculations yield to average composition of 12.29-12.43 wt. $\% \mathrm{Al}_{2} \mathrm{O}_{3}, 0.33-0.37$ wt. $\% \mathrm{TiO}_{2}$ and $0.96 \mathrm{FeO} / \mathrm{MgO}$ ratio for the parental melt. This composition overlaps those of magmas with boninitic affinity, which are Ti-poor $(<0.5$ wt. $\% \mathrm{TiO}_{2}$ ) with low $\mathrm{FeO} / \mathrm{MgO}$ ratios $(>0.8$ wt. \% MgO) (Le Bas, 2000). Boninite melts have 


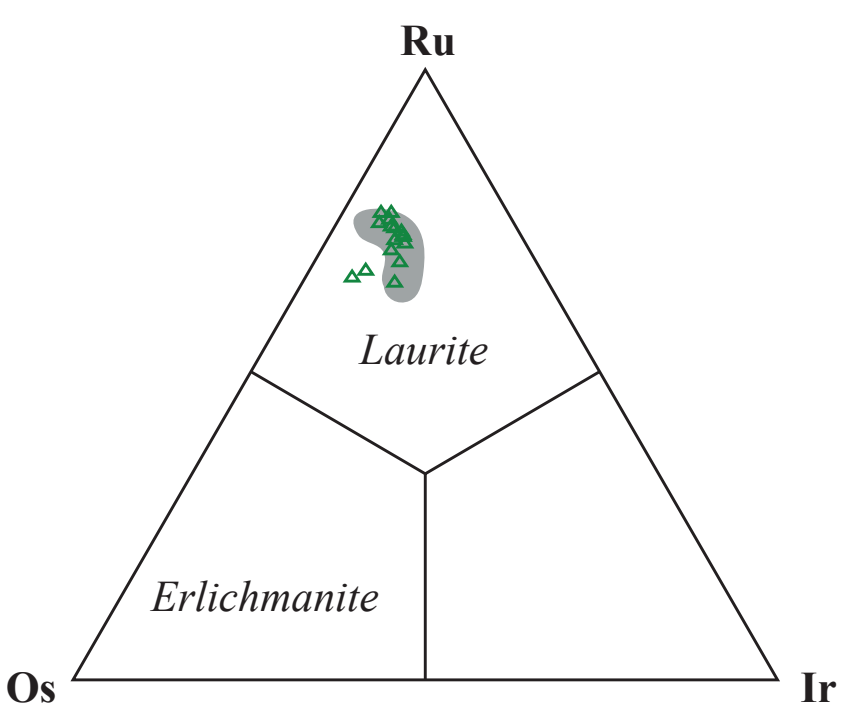

Laurite from chromitite bodies from Havana-

Matanzas Ophiolite (from Llanes-Castro et al., 2015)

Figure 6 Composition (at.\%) of laurite grains from the chromitite veins.

relatively high $\mathrm{SiO}_{2}$ contents and are normally saturated in orthopyroxene (Falloon and Danyushevsky, 2000, and references therein). Therefore, we consider that the chromitite veins from the Havana-Matanzas Ophiolite formed from low-Ti high- $\mathrm{Mg}$ andesitic melts with boninitic affinity that originated in the fore-arc of an intra-oceanic island arc. This interpretation is in accordance with models of formation of high-Cr ophiolitic chromitites by melt-rock reaction processes (e.g., Zhou and Robinson, 1997; Melcher et al., 1997; Proenza et al. 1999; González-Jiménez et al., 2014, and references therein). On the other hand, it has been proposed that two olivine-saturated liquids, one residual to dunite and the other extracted from harzburgite at higher pressure, could mingle to produce chromite-saturated hybrid melts from which chromitite can form (Ballhaus, 1998). This has been demonstrated by thermodynamic modeling (Abuamarah et al., 2020).

In the case of the Havana-Matanzas Ophiolite chromitite veins, we interpret the systematic occurrence of the chromitite within orthopyroxenite and the lack of chromitite compositional variations towards their contact as evidence for the contemporaneous formation of the two types of rock. We infer that a $\mathrm{Mg}$-rich andesitic melt with boninitic affinity interacted with harzburgite upon infiltration, triggering the peritectic reaction $\mathrm{Ol}+\mathrm{Liquid}=\mathrm{Opx}+\mathrm{Chr}$ that leaded to consumption of olivine and precipitation of orthopyroxene and chromite (Kelemen et al., 1992, 1995; Wang et al., 2016). Further infiltration of new batches of liquid would mix with the residual liquids to yield secondary chromite-saturated melt which would precipitate chromite to form the chromitite veins (Irvine, 1977). The small volume of the chromitite veins indicates that the volume of parental magma required for their formation is lower than for the rest of chromitite bodies from the Havana-Matanzas Ophiolite.

\subsubsection{PGE MINERALIZATIONS AND THEIR GENETIC IMPLICATIONS}

As mentioned above, high-Cr chromitites are interpreted to crystallize from magmas with boninitic affinity. These magmas are S-undersaturated and normally contain higher PGE contents than tholeiitic magmas, hence explaining the PGE-rich composition of high-Cr chromitites relative to high-Al chromitites (e.g., Peck et al., 1992; Zhou et al., 1998; Saha et al., 2015, and references therein). In the Havana-Matanzas Ophiolite chromitite veins, the abundant PGM inclusions reflect the PGE-rich composition expected for high-Cr chromitites. The main PGM phase found in the studied chromitites is laurite, which is also the main PGM observed in other ophiolitic chromitites worldwide (e.g., Luguet et al., 2007; González-Jiménez et al., 2014, and references therein). This PGE mineralogy also reflects the IPGE (Os, Ir, Ru) enrichment relative to PPGE (Rh, Pt and Pd) typical for mantle-hosted ophiolitic chromitites (e.g., Zhou et al., 1996; Ahmed and Arai, 2002).

On the basis of their textural features and composition, the PGM identified in the chromitite veins can be divided into primary and secondary phases. Laurite crystals with homogeneous compositions or displaying magmatic textures (crystal growth bands and oscillatory zoning; Figures 4C to $4 \mathrm{~F}$ ) are considered primary. In contrast, those 
PGM grains displaying rugged surfaces or that overgrow the surface of primary laurite (Figures $4 \mathrm{~L}$ to $4 \mathrm{~N}$ ) are interpreted as secondary in origin, very likely originated by alteration of pre-existing PGM initially included in chromite and by local remobilization and redeposition of PGE during serpentinization (e.g., Garuti and Zaccarini, 1997; Proenza et al., 2008). Regarding primary laurite grains, most authors agree that they form at high-temperature stages from S-undersaturated mafic melts before or coeval with crystallization of chromite. In the studied samples, their predominantly euhedral morphology and their location in unaltered chromite suggests that they crystallized from a magma and were entrapped by growing grains of chromite (e.g., Melcher et al., 1997; González-Jiménez et al., 2009).

As mentioned above, the composition of the laurite inclusions from the Havana-Matanzas Ophiolite chromitite veins is generally Os-rich (Table 4). The $\mathrm{Ru}^{-} \mathrm{Os}$ contents of laurite are strongly influenced by sulfur fugacity $\left(f \mathrm{~S}_{2}\right)$ and temperature (e.g., Brenan and Andrews, 2001; Andrews and Brenan, 2002): the solubility of Os in laurite increases with decreasing temperature and/or increasing $f \mathrm{~S}_{2}$ (e.g., González-Jiménez et al., 2009). Nearly stoichiometric laurite $\left(\operatorname{RuS}_{2}\right)$ has a maximum temperature of crystallization around $1200^{\circ} \mathrm{C}$, at very low sulfur fugacity $\left(\log f \mathrm{~S}_{2}<-2\right.$; Brenan and Andrews, 2001; Andrews and Brenan, 2002). Therefore, the Os-rich composition of laurite from the Havana-Matanzas Ophiolite chromitite veins indicates that they probably crystallized at relatively lower temperatures and/or higher $f$ $\mathrm{S}_{2}$ than stoichiometric laurite. Lower temperatures for the Os-rich laurite formation would be in accordance with the cooling temperatures calculated for the orthopyroxenite $\left(700-1100^{\circ} \mathrm{C}\right)$, which formed contemporaneously with the chromitite veins containing the PGM. Moreover, the oscillatory zoning displayed by some laurite crystals (Figure 4F), with $\mathrm{Ru}-\mathrm{Os}$ variations, lead to suggest that the PGM crystallized within a system dominated by short-term variations of $f \mathrm{~S}_{2}$ and temperature (González-Jiménez et al., 2009). That could be the case of the model proposed for the chromitite formation, in which variations in the physicochemical properties of the parental melt would be a natural consequence of the melt-rock reactions. The chromite-bearing melts from the proposed model could efficiently concentrate high amounts of PGE along with already nucleated chromite. In this context, PGM tend to wet and/ or nucleate along the edges of growing chromite (e.g., Finnigan et al., 2008, and references therein). Therefore, chromite would act as a PGM collector, favoring the PGM concentration process. The efficiency of the mechanical collection is maximized when the magma crystallizing the PGM is in contact with small volumes of chromite (Augé et al., 2005). For this reason, the small and thin chromitite veins from the Havana-Matanzas Ophiolite show extreme enrichment in PGM inclusions.

\section{Conclusions}

Orthopyroxenite bands within the Havana-Matanzas Ophiolite harzburgites formed from magmas with boninitic affinity, which are typical of fore-arc settings in intra-oceanic arcs. Small and thin PGM-rich chromitite veins are hosted within the orthopyroxenite. The chromitites are in equilibrium with the host orthopyroxenite and they also show boninitic affinity. We propose that the orthopyroxenite and the chromitite are formed contemporaneously after the reaction between pre-existing harzburgite and a Si-rich melt, probably of boninitic affinity. This reaction generated orthopyroxenite and a secondary melt that would mix with newly infiltrated liquid to form a Cr-saturated liquid from which chromite would precipitate to form chromitite veins within the orthopyroxenite. The growing chromite would act as a PGM collector due to the PGE affinity for chromite. The small volume of chromitite formed would maximize the efficiency of the mechanical collection, resulting in the high abundance of primary PGM inclusions observed in the chromitite veins from the Havana-Matanzas Ophiolite. 


\section{References}

Abuamarah, B., Asimow, P.D., Azer, M.K., Ghrefat, H., 2020, Suprasubductionzone origin of the podiform chromitites of the Bir Tuluhah, Saudi Arabia, during Neoproterozoic assembly of the Arabian Shield: Lithos, 360-361, 105439. https:// doi.org/10.1016/j.lithos.2020.105439

Ahmed, A., Arai, S., 2002, Unexpectedly high-PGE chromitite from the deeper mantle section of the northern Oman ophiolite and its tectonic implications: Contributions to Mineralogy and Petrology, 143(3), 263-278. https://doi. org/10.1007/s00410-002-0347-8

Andrews, D.R.A., Brenan, J.M., 2002, Phaseequilibrium constraints on the magmatic origin of laurite + Ru-Os-Ir alloy: Canadian Mineralogist, 40, 1705-1716. https://doi. org/10.2113/gscanmin.40.6.1705

Arai, S., 1992, Chemistry of chromian spinel in volcanic rocks as a potential guide to magma chemistry: Mineralogical Magazine, 56, 173-184. https://doi.org/10.1180/ minmag. 1992.056.383.04

Arai, S., Miura, M., 2016, Formation and modification of chromitites in the mantle: Lithos, 264, 277-295. https://doi. org/10.1016/j.lithos.2016.08.039

Arai, S., Uesugi, J., Ahmed, A.H., 2004, Upper crustal podiform chromitite from the northern Oman ophiolite as the stratigraphically shallowest chromitite in ophiolite and its implication for $\mathrm{Cr}$ concentration: Contributions to Mineralogy and Petrology, 147, 145-154. https://doi.org/10.1007/ s00410-004-0552-8

Augé, T., Genna, A., Legendre, O., 2005, Primary platinum mineralization in the Nizhny Tagil and Kachkanar ultramafic complexes, Urals, Russia: a genetic model for PGE concentration in chromite-rich zones: Economic Geology, 100, 707-732. https:// doi.org/10.2113/gsecongeo.100.4.707

Ballhaus, C., 1998, Origin of podiform chromite deposits by magma mingling:
Earth and Planetary Science Letters 156, 185-193. https://doi.org/10.1016/ s0012-821x(98)00005-3

Bédard, J.H., Hébert, R., 1998, Formation of chromitites by assimilation of crustal pyroxenites and gabbros into peridotitic intrusions: North Arm Mountain massif, Bay of Islands ophiolite, Newfoundland, Canada: Journal of Geophysical Research, 103(B3), 5165-5184. https://doi. org/10.1029/97JB03291

Berly, T.J., Hermann, J., Arculus, R.J., Lapierre, H., 2006, Supra-subduction zone pyroxenites from San Jorge and Santa Isabel (Solomon Islands): Journal of Petrology, 47, 15311555. https://doi.org/10.1093/petrology/ egl019

Bonavia, F.F., Diella, V., Ferrario, A., 1993, Precambrian podiform chromitites from Kenticha Hill, southern Ethiopia: Economic Geology, 88(1), 198-202. https://doi. org/10.2113/gsecongeo.88.1.198

Brenan, J.M., Andrews, D., 2001, Hightemperature stability of laurite and $\mathrm{Ru}-\mathrm{Os}$ Ir alloy and their role in PGE fractionation in mafic magmas: Canadian Mineralogist, 39, 341-360. https://doi.org/10.2113/ gscanmin.39.2.341

Brey, G.P., Köhler, T., 1990, Geothermobarometry in four-phase lherzolites II. New thermobarometers, and practical assessment of existing thermobarometers: Journal of Petrology, 31(6), 1353-1378. https://doi. org/10.1093/petrology/31.6.1353

Dick, H.J.B., Bullen, T., 1984, Chromian spinel as a petrogenetic indicator in abyssal and alpinetype peridotites and spatially associated lavas: Contributions to Mineralogy and Petrology, 86, 54-76. https://doi.org/10.1007/ BF00373711

Falloon, T.J., Danyushevsky, L.V., 2000, Melting of refractory mantle at $1.5-2$ and $2.5 \mathrm{GPa}$ under anhydrous condition and $\mathrm{H}_{2} \mathrm{O}$ undersaturated conditions: implications for the petrogenesis of high-Ca boninites and the influence of subduction components 
on mantle melting: Journal of Petrology, 41, 257-283. https://doi.org/10.1093/ petrology/41.2.257

Ferrario, A., Garuti, G., 1987, Platinum-group minerals in chromite-rich horizons of the Niquelandia complex (central Goias, Brazil) in Prichard H.M., Potts P.J., Bowles J.F.W., and Cribb S.(eds.), Geo-Platinum 87: London, U.K., Elsevier Applied Science, 261-272.

Finnigan, C.S., Brenan, J.M., Mungall, J.E., McDonough, W.F., 2008, Experiments and models bearing on the role of chromite as a collector of platinum group minerals by local reduction: Journal of Petrology, 49, 16471665. https://doi.org/10.1093/petrology/ egn041

Fonseca, E., Zelepugin, V.N., Heredia, M., 1985, Structure of the ophiolite association of Cuba: Geotectonic, 1, 321-329.

Garcia-Casco, A., Torres-Roldán, R.L., IturraldeVinent, M.A., Millán, G., Núñez Cambra, K., Lázaro, G., Rodríguez Vega, A., 2006, High pressure metamorphism of ophiolites in Cuba: Geologica Acta, 4(1-2), 63-88. https://doi.org/10.1344/105.000000358

Garcia-Casco, A., Iturralde-Vinent, M.A, Pindell, J., 2008, Latest Cretaceous collision/ accretion between the Caribbean Plate and Caribeana: Origin of metamorphic terranes in the Greater Antilles: International Geology Review, 50, 781- 809. https://doi. org/10.2747/0020-6814.50.9.781

Garuti, G., Zaccarini, F., 1997, In situ alteration of platinum-group minerals at low temperature: evidence from serpentinised and weathered chromitite of the Vourinos complex, Greece: The Canadian Mineralogist 35, 611-626.

Gervilla, F., González-Jiménez, J.M., Hidas, K., Marchesi, G., Piña, R., 2019, Geology and metallogeny of the upper mantle rocks from the Serranía de Ronda: España, Editorial La Serranía y Sociedad Española de Mineralogía, $124 \mathrm{p}$.

Gervilla, F., Proenza, J.A., Frei, R., GonzálezJiménez, J.M., Garrido, C.J., Melgarejo,
J.C., Meibom, A., Díaz-Martínez, R., Lavaut, W., 2005, Distribution of platinumgroup elements and Os isotopes in chromite ores from Mayarí-Baracoa Ophiolilte Belt (eastern Cuba): Contributions to Mineralogy and Petrology 150, 589-607. https://doi. org/10.1007/s00410-005-0039-2

González-Jiménez, J.M., Gervilla, F., Proenza, J.A., Kerestedjian, T., Augé, T., Bailly, L., 2009, Zoning of laurite $\left(\mathrm{RuS}_{2}\right)$ - erlichmenite $\left(\mathrm{OsS}_{2}\right)$ : implications for the origin of PGM in ophiolite chromitites: European Journal of Mineralogy, 21, 419-432. https://doi. org/10.1127/0935-1221/2009/0021-1921

González-Jiménez,J.M., Proenza,J.A., Gervilla, F., Melgarejo, J.G., Blanco-Moreno, J.A., RuizSánchez, R., Griffin, W.L., 201 la, High-Cr and high-Al chromitites from the Sagua de Tánamo district, Mayarí-Cristal ophiolitic massif (eastern Cuba): Constraints on their origin from mineralogy and geochemistry of chromian spinel and platinum-group elements: Lithos, 125, 101-121. https://doi. org/10.1016/j.lithos.2011.01.016

González-Jiménez, J.M., Augé, T., Gervilla, F., Bailly, L., Proenza, J.A., Griffin, W.L., $2011 \mathrm{~b}$, Mineralogy and geochemistry of platinum-rich chromitites from the mantlecrust transition zone at Ouen Island, New Caledonia ophiolite: The Canadian Mineralogist, 49, 1549-1570. https://doi. org/10.3749/canmin.49.6.1549

González-Jiménez, J.M., Marchesi, C., Griffin, W.L., Gutiérrez-Narbona, R., Lorand, J.P., O'Reilly, S.Y., Garrido, C.J., Gervilla, F., Pearson, N.J., Hidas, K., 2013, Transfer of Os isotopic signatures from peridotite to chromitite in the subcontinental mantle: insights from in situ analysis of platinumgroup and base-metal minerals (Ojén peridotite massif, southern Spain): Lithos 164-167, 74-85. https://doi.org/10.1016/j. lithos.2012.07.009

Gonzalez-Jimenez, J.M., Griffin, W.L., Proenza, J.A., Gervilla, F., O'Reilly, S.Y., Akbulut, M., Pearson, N.J., Arai, S., 2014, Chromitites in 
ophiolites: How, where, when, why? Part II. The crystallization of chromitites: Lithos, 189, 140-158. https://doi.org/10.1016/j. lithos.2013.09.008

Grant, T.B., Harlov, D.E., Rhede, D., 2016, Experimental formation of pyroxenite veins by reactions between olivine and $\mathrm{Si}, \mathrm{Al}, \mathrm{Ca}$, $\mathrm{Na}$, and Cl-rich fluids at $800{ }^{\circ} \mathrm{C}$ and 800 MPa: Implications for fluid metasomatism in the mantle wedge: American Mineralogist, 101, 808-818. https://doi.org/10.2138/ am-2016-5441

Hellebrand, E., Snow, J.E., Dick, H.J., Hofmann, A.W., 2001, Coupled major and trace elements as indicators of the extent of melting in midocean-ridge peridotites: Nature, 410, 677681. https://doi.org/10.1038/35070546

Hirauchi, K.I., Tamura, A., Arai, S., Yamaguchi, H., Hisada, K.I., 2008, Fertile abyssal peridotites within the Franciscan subduction complex, central California: Possible origin as detached remnants of oceanic fracture zones located close to a slow-spreading ridge: Lithos, 105, 319-328. https://doi. org/10.1016/j.lithos.2008.05.002

Irvine, T. N, 1977, Origin of chromitite layers in the Muskox intrusion and other stratiform intrusions: A new interpretation: Geology, 5, 273-277. https://doi.org/10.1130/00917613(1977)5<273:OOCLIT>2.0.CO;2

Ishii, T., Robinson, P.T., Maekawa, H., Fiske, R., 1992, Petrological studies of peridotites from diapiric serpentinite seamounts in the Izu-Ogasawara-Mariana forearc, in Fryer P., Pearce J.A., Stokking L.B. (eds.), Proceedings of the Ocean Drilling Program Scientific Results 125, Texas, USA, Ocean Drilling Program and College Station, 445-485.

Iturralde-Vinent, M.A., 1996, Geología de las ofiolitas de Cuba: in, Iturralde-Vinent, M.A. (Ed.), Ofiolitas y arcos volcánicos de Cuba, IGCP Project 364, Special Contribution, 1, 83-120.

Iturralde-Vinent, M. A., 1998, Sinopsis de la constitución geológica de Cuba: Acta Geológica Hispánica, 33, 9-56.
Iturralde-Vinent, M.A., Díaz Otero, G., GarcíaCasco, A., Van Hinsbergen, D.J.J. 2008, Paleogene Foredeep Basin Deposits of North-Central Cuba: A Record of ArcContinent Collision between the Caribbean and North American Plates: International Geology Review, 50, 863-884. https://doi. org/10.2747/0020-6814.50.10.863

Iturralde-Vinent, M.A., Garcia-Casco, A., RojasAgramonte, Y., Proenza, J.A., Murphy, J.B., Stern, R. J., 2016, The geology of Cuba: A brief overview and synthesis: GSA Today, 26, 4-10. https://doi.org/10.1130/ GSATG296A.1

Kelemen, P.B., 1990, Reaction between ultramafic wall rock and fractionating basaltic magma. I. Phase relations, the origin of calcalkaline magma series, and the formation of discordant dunite: Journal of Petrology, 31, 51-98. https://doi.org/10.1093/ petrology/31.1.51

Kelemen, P.B., Dick, H.J.B., Quick, J.E., 1992, Formation of harzburgite by pervasive melt/rock reaction in the upper mantle: Nature, 358, 635-641. https://doi. org/10.1038/358635a0

Kelemen, P.B., Shimizu, N., Salters, W.J.W., 1995, Extraction of mid-ocean ridge basalts from the upwelling mantle by focused flow of melt in dunite channels: Nature 375, 747-753. https://doi.org/10.1038/375747a0

Kerr, A. G., Iturralde-Vinent, M. A., Saunders, A. D., Babbs, T. L., Tarney, J., 1999, A new plate tectonic model of the Caribbean: Implications from a geochemical reconnaissance of Cuban Mesozoic volcanic rocks: GSA Bulletin, 111, 1581-1599. https://doi.org/10.1130/00167606(1999)111<1581:ANPTMO>2.3. $\mathrm{CO} ; 2$

Köhler, T. P., Brey, G. P., 1990, Calcium exchange between olivine and clinopyroxene calibrated as a geothermobarometer for natural peridotites from 2 to $60 \mathrm{~kb}$ with applications: Geochimica et Cosmochimica Acta, 54, 2375-2388. https://doi. org/10.1016/0016-7037(90)90226-B 
Le Bas, M.J., 2000, IUGS reclassification of the high-Mg and picritic volcanic rocks: Journal of Petrology, 41, 1467-1470. https://doi. org/10.1093/petrology/41.10.1467.

Leblanc, M., Nicolas, A., 1992, Les chromitites ophiolitiques : Chronique de la Recherche Minière, 507, 3-25.

Lewis, J. F., Draper, G., Proenza, J. A., Espaillat, J., Jiménez,J., 2006, Ophiolite-related ultramafic rocks (serpentinites) in the Caribbean region: a review of their occurrence, composition, origin, emplacement and Ni-laterite soil formation: Geologica Acta, 4, 237-263. https://doi.org/10.1344/105.000000368

Llanes-Castro, A.I., Proenza, J.A., Zaccarini, F., Garuti, G., Pacheco-Sarlabous, M.S.C., 2015, Al-and Cr-rich chromitites from the Eastern Havana-Matanzas ophiolites (Western Cuba): Episodes, 38, 334-343. https://doi.org/10.18814/epiugs/2015/ v38i4/82429

Llanes-Castro, A.I., Cruz-Gámez, E.M., PérezRodriguez, M., López-Cruz, O., Furnes, H., 2019, Petrogenesis of plagiogranite and associated diorites and mafic rocks in the Habana-Matanzas ophiolites, northwestern half of central Cuba: Journal of the Geological Society, 176, 992-1006. https:// doi.org/10.1144/jgs2018-116

Luguet, A., Shirey, S.B., Lorand, J.P., Horan, M.F., Carlson, R.W., 2007, Residual platinumgroup minerals from highly depleted harzburgites of the Lherz massif (France) and their role in HSE fractionation of the mantle: Geochimica et Cosmochimica Acta, 71, 3082-3097. https://doi.org/10.1016/j. gca.2007.04.011

Melcher, F., Meisel, T., 2004, A metamorphosed early Cambrian crust-mantle transition in the Eastern Alps, Austria: Journal of Petrology, 45, 1689-1723. https://doi.org/10.1093/ petrology/egh030

Melcher, F., Grum, W., Simon, G., Thalhammer, T. V., Stumpfl, E. F., 1997, Petrogenesis of the ophiolitic giant chromite deposits of
Kempirsai, Kazakhstan: a study of solid and fluid inclusions in chromite: Journal of Petrology, 38, 1419-1458. https://doi. org/10.1093/petroj/38.10.1419

Pagé, P., Bédard, J. H., Schroetter, J. M., Tremblay, A., 2008, Mantle petrology and mineralogy of the Thetford Mines Ophiolite Complex: Lithos, 100, 255-292. https://doi. org/10.1016/j.lithos.2007.06.017

Peck, D.C., Keays, R.R., Ford, R.J., 1992, Direct crystallization of refractory platinum group element alloys from boninitic magmas: Evidence from western Tasmania: Australian Journal of Earth Sciences, 39, 373-387. https://doi. org/10.1080/08120099208728031

Proenza, J., Gervilla, F., Melgarejo, J. C., Bodinier, J. L., 1999, Al-and Cr-rich chromitites from the Mayari-Baracoa ophiolitic belt (eastern Cuba); consequence of interaction between volatile-rich melts and peridotites in suprasubduction mantle: Economic Geology, 94, 547-566. https://doi.org/10.2113/ gsecongeo.94.4.547

Proenza, J.A., Zaccarini, F., Cábana, G., Escayola, M., Shalamu, K.A., Garuti, G., 2008, Chromite composition and platinum-group minerals in chromitites of the western ophiolitic belt from Pampeans Ranges of Córdoba, Argentina: Ore Geology Reviews, 33, 32-48. https://doi.org/10.1016/j. oregeorev.2006.05.009

Rojas-Agramonte, Y., Kröner, A., Garcia-Casco, A., Kemp, T., Hegner, E., Pérez, M., Barth, M., Liu, D., Fonseca-Montero, A., 2010, Zircon ages, Sr-Nd-Hf isotopic compositions, and geochemistry of granitoids associated with the northern ophiolite mélange of Central Cuba: Tectonic implication for Late Cretaceous magmatism in the Northwestern Caribbean: American Journal of Science, 310, 1453-1479. https://doi. org/10.2475/10.2010.09

Saha, A., Manikyamba, C., Santosh, M., Ganguly, S., Khelen, A.C., Subramanyam, K.S.V., 
2015, Platinum Group Elements (PGE) geochemistry of komatiites and boninites from Dharwar Craton, India: Implications for mantle melting processes: Journal of Asian Earth Sciences, 105, 300-319. https:// doi.org/10.1016/j.jseaes.2015.01.020

Varfalvy, V., Hébert, R., Bédard, J. H., Laflèche, M. R., 1997, Petrology and geochemistry of pyroxenite dykes in upper mantle peridotites of the North Arm Mountain Massif, Bay of Islands Ophiolite, Newfoundland; implications for the genesis of boninitic and related magmas: The Canadian Mineralogist, 35, 543-570.

Wang, C., Liang, Y., Dygert, N., Xu, W., 2016, Formation of orthopyroxenite by reaction between peridotite and hydrous basaltic melt: an experimental study: Contributions to Mineralogy and Petrology 171, 77. https:// doi.org/10.1007/s00410-016-1287-z

Whattam, S. A., Stern, R.J., 2011, The 'subduction initiation rule': a key for linking ophiolites, intra-oceanic forearcs, and subduction initiation: Contributions to Mineralogy and Petrology, 162, 1031-1045. https://doi. org/10.1007/s00410-011-0638-z
Zaccarini, F., Garuti, G., Proenza, J. A., Campos, L., Thalhammer, O. A. R., Aiglsperger, T., Lewis, J. F., 2011, Chromite and platinum group elements mineralization in the Santa Elena Ultramafic Nappe (Costa Rica): geodynamic implications: Geologica Acta, 9, 407-423. https://doi. org/10.1344/105.000001696

Zhou, M.F., Robinson, P.T., 1997, Origin and tectonic environment of podiform chromite deposits: Economic Geology, 92, 259-262. https://doi.org/10.2113/ gsecongeo.92.2.259

Zhou, M.F., Robinson, P.T., Malpas, J., Zijin, L., 1996, Podiform chromites in the Luobusa Ophiolite (Southern Tibet): implications for melt-rock interaction and chromite segregation in the upper mantle: Journal of Petrology 37, 3-21.

Zhou, M.F., Sun, M., Keays, R.R., Kerrich, R.W., 1998, Controls on platinum-group elemental distributions of podiform chromitites: a case study of high-Cr and high-Al chromitites from Chinese orogenic belts: Geochimica et Cosmochimica Acta, 62, 677-688. https:// doi.org/10.1016/S0016-7037(97)00382-7 